\title{
Systematic investigation of three-nucleon force effects in elastic scattering of polarized protons from deuterons at intermediate energies
}

\author{
K. Ermisch, ${ }^{1, *}$ H. R. Amir-Ahmadi, ${ }^{1}$ A. M. van den Berg, ${ }^{1}$ R. Castelijns, ${ }^{1}$ B. Davids,,${ }^{1}$ A. Deltuva,,${ }^{2}$ E. Epelbaum, ${ }^{3}$ \\ W. Glöckle, ${ }^{4}$ J. Golak, ${ }^{5}$ M. N. Harakeh, ${ }^{1}$ M. Hunyadi, ${ }^{1}$ M. A. de Huu, ${ }^{1}$ N. Kalantar-Nayestanaki, ${ }^{1, \dagger}$ H. Kamada, ${ }^{6}$ \\ M. Kišs, ${ }^{1}$ M. Mahjour-Shafiei, ${ }^{1}$ A. Nogga, ${ }^{7}$ P. U. Sauer, ${ }^{2}$ R. Skibiński,${ }^{5}$ H. Witała, ${ }^{5}$ and H. J. Wörtche ${ }^{1}$ \\ ${ }^{1}$ Kernfysisch Versneller Instituut (KVI), Groningen, The Netherlands \\ ${ }^{2}$ Institut für Theoretische Physik, Universität Hannover, Germany \\ ${ }^{3}$ Thomas Jefferson National Accelerator Laboratory, Newport News, Virginia 23606, USA \\ ${ }^{4}$ Institut für Theoretische Physik II, Universität Bochum, Germany \\ ${ }^{5}$ Institute of Physics, Jagellonian University, Cracow, Poland \\ ${ }^{6}$ Department of Physics, Faculty of Engineering, Kyushu Institute of Technology, Kitakyushu, Japan \\ ${ }^{7}$ Institute for Nuclear Theory, University of Washington, Seattle, Washington 98195, USA
}

(Received 15 May 2004; published 28 June 2005)

\begin{abstract}
The question, whether the high-quality nucleon-nucleon potentials can successfully describe the three-nucleon system, and to what extent three-nucleon forces ( $3 N F$ s) play a role, has become very important in nuclear few-body physics. One kinematic region where effects because of $3 N F$ s show up is in the minimum of the differential cross section of elastic nucleon-deuteron scattering. Another observable, which could give an indication about the contribution of the spin to $3 N F$ s, is the vector analyzing power. To investigate the importance of $3 N F s$ systematically over a broad range of intermediate energies, both observables of elastic proton-deuteron scattering have been measured at proton bombarding energies of 108, 120,135, 150,170, and $190 \mathrm{MeV}$, covering an angular range in the center-of-mass system between $30^{\circ}$ and $170^{\circ}$. The results show unambiguously the shortcomings of calculations employing only two-body forces and the necessity of the inclusion of 3 NFs. They also show the limitations of the results of the present day models for few-nucleon systems at backward angles, especially at higher beam energies. New calculations based on chiral perturbation theory are also presented and compared with the data at the lowest energy.
\end{abstract}

DOI: 10.1103/PhysRevC.71.064004

PACS number(s): 13.75.Cs, 21.30.-x, 21.45.+v, 25.10.+s

\section{INTRODUCTION}

In the past few years, several semiphenomenological two-nucleon models, CD-Bonn, Argonne-V18 (AV18), Nijmegen-I, Nijmegen-II, and Reid93 [1-5], have become available, which describe two-nucleon scattering observables accurately. Furthermore, because of the availability of supercomputers, Faddeev-type three-nucleon calculations have become feasible. The use of the modern two-nucleon potentials, to describe three-nucleon scattering observables, leads to various degrees of agreement between the calculations and the experimental data, depending on the observable being studied [6]. At low incident beam energies, up to $\approx 30 \mathrm{MeV}$, the differential cross section of nucleon-deuteron scattering is described rather well using solely two-nucleon potentials. In contrast, the description of the analyzing power has failed and the inclusion of three-nucleon forces ( $3 N F s$ ) into the calculations has not remedied the discrepancy, leading to the well-known $A_{y}$ puzzle. Also for low energies, tensor-analyzing powers and spin-transfer coefficients are rather well described using solely $N N$ forces [6], whereas at intermediate and higher energies the inclusion of $3 N$ forces is necessary [7-9].

\footnotetext{
* Present address: Onsala Observatory, Chalmers University of Technology, Gothenburg, Sweden.

†Corresponding author: nasser@kvi.nl
}

Calculations have shown that effects because of $3 \mathrm{NF}$ should clearly manifest themselves in the minimum of the differential cross section at incident-beam energies $\gtrsim 65 \mathrm{MeV} /$ nucleon $[10,11]$. Although the contribution of the $3 N F$ to the differential cross section is rather constant across the center-of-mass (c.m.) angular range, the contribution from pure two-nucleon interactions has a pronounced dip around $90^{\circ}$ c.m. angle and decreases relative to the contribution of the $3 N F$ with increasing incident-beam energy. Measurements of elastic proton-deuteron scattering at $65 \mathrm{MeV} /$ nucleon [12] showed indeed that the use of two-nucleon potentials exclusively does not suffice to describe the differential cross section at this energy and the inclusion of a modern $3 \mathrm{NF}$ was necessary. Older measurements on the proton-deuteron system, conducted at energies of around $150 \mathrm{MeV} /$ nucleon [13,14], $181 \mathrm{MeV} /$ nucleon [15], and $198 \mathrm{MeV} /$ nucleon [16] either lacked precision and/or covered only part of the centerof-mass angular scattering range, making it difficult to make a systematic study of the effect of the $3 \mathrm{NF}$.

Another observable, which should give an indication about the role played by the spin degree of freedom in $3 \mathrm{NF}$, is the vector analyzing power $A_{y}$. As mentioned before, calculations with and without $3 N F$ give contradictory results for this observable at low bombarding energies. At higher bombarding energies, several measurements exist at $120 \mathrm{MeV} /$ nucleon [17] and around $150 \mathrm{MeV} /$ nucleon [13, 14,18] and $190 \mathrm{MeV} /$ nucleon [15,16,18,19]. Furthermore, 
high-precision measurements for selected angles exist at several energies [20]. Recently, systematic investigations of this observable at four incident-beam energies were published [21]. At each energy, measurements were done at $30^{\circ} \leqslant \theta_{\text {c.m. }} \leqslant$ $170^{\circ}$.

In this article, measurements will be presented for cross sections and analyzing powers for proton beam energies of $108,120,135,150,170$, and $190 \mathrm{MeV}$ and an angular range of $30^{\circ}$ to $170^{\circ}$. The present data set will serve as a consistent one at intermediate energies for the investigation of the effects of the $3 N F$. Parts of the data have already been published [21,22]. The data will be compared with two sets of calculations that take the effect of $N N$ forces and $3 N F$ into account. This article is organized as follows. In Sec. II, the general ingredients of the theoretical calculations are presented. The experimental setup is laid out in some detail in Sec. III. The analysis techniques are briefly presented in Sec. IV. The experimental results are discussed in Sec. V along with the results of various calculations. A summary of the work can be found in Sec. VI.

\section{THEORETICAL FRAMEWORK}

The theoretical approaches are twofold. If one works in a Hilbert space with only nucleonic degrees of freedom, the $3 \mathrm{~N}$ forces can by their very definition not be broken up into a sequence of pair forces. However, if one works in an extended Hilbert space, allowing the nucleon to be excited into the $\Delta$, transitions take place and a process like the $2 \pi$-exchange between 3 nucleons with an intermediate $\Delta$ can be reduced to a sequence of pair potentials with an intermediate free propagation of 2 nucleons and one $\Delta$. In the first scenario, with nucleon degrees of freedom only, this process is irreducible and appears as an instantaneous $3 N$ force. We shall show results for both approaches. The first one will be briefly described in Sec. II A and the second one in Sec. II B. A new formulation based on effective field theory constrained by chiral symmetry belongs to the first approach and will be also applied in this article. It is briefly addressed in Sec. II A.

\section{A. The $3 N$ Hilbert Space Approach}

Three-nucleon scattering based on $N N$ and $3 N$ forces can be formulated in the Faddeev scheme in various manners [6]. We follow the formulation given in [23], where like for the $3 N$ bound state [24] only one equation has to be solved. That equation determines part of the breakup amplitude out of which the physical breakup amplitude and the amplitude for elastic scattering follows by quadrature. The solution is carried through in momentum space and in a partial-wave decomposition, which leads to a coupled set of integral equations in two variables, the magnitudes of the two relative Jacobi momenta. For technical details we refer the reader to Ref. [25,26]. The intermediate energies investigated in this article require a rather large number of angular momentum states for the underlying $N N$ system and the total $3 N$ angular momenta. We kept the $N N$ force up to total $N N$ angular momentum $j=5$. This turned out to be sufficient as the comparison with results for $j=6$ revealed. The total $3 N$ angular momenta $J$ were kept up to $J=25 / 2$ and the action of the $3 N$ force up to $J=13 / 2$, which guaranteed sufficient convergence. We use the $2 N$ forces AV18, NijmI, and NijmII, and CD-Bonn. The resulting $3 N$ observables are rather similar to each other and are combined into a narrow band in the figures presented in the article. This is an important observation, because it allows to clearly distinguish modifications in the observables resulting from the additional action of $3 N$ forces from the spread caused by different $N N$ forces only. Together with each of the $N N$ forces used, we adjusted the $2 \pi$-exchange Tucson Melbourne 3NF TM99 [27] to the ${ }^{3} \mathrm{H}$ binding energy [28]. This was achieved by one parameter in that force, the strong form factor cutoff value. The predictions of these $N N$ forces with the TM99 $3 N$ force are also combined in a second band in the figures presented. The Urbana IX $3 N$ force was taken together with AV18 and guarantees the correct ${ }^{3} \mathrm{H}$ binding energy by its very construction [29].

The TM99 $3 N$ force relies on a low momentum expansion of the off-shell $\pi N$ scattering amplitude, which is a modification of the original version [30] removing a term which was in conflict with chiral symmetry [31]. This force also incorporates, among other dynamical ingredients, the effect of an intermediate (static) $\Delta$ but goes beyond that and includes also $s$-wave contributions in the $\pi N$ system.

The Urbana IX $3 N$ force is a pure $2 \pi$-exchange with an intermediate static $\Delta$ but supplemented by a spin and isospin independent purely phenomenological short-range part. There are two overall constants in this force that have been adjusted to the ${ }^{3} \mathrm{H}$ binding energy and the density of nuclear matter [29].

In addition to these conventional dynamical ingredients, we applied newly developed nuclear forces based on effectivefield theory constrained by chiral symmetry $[32,33]$. We show here results based on the forces up to the next-to-next leading order (NNLO) where, for the first time, nonvanishing $3 N$ forces appear $[34,35]$. They occur in three topologies, a $2 \pi$-exchange, a one $\pi$-exchange between a $N N$ contact force and the third nucleon, and a pure $3 N$ contact force. Each of the latter two processes depend on one unknown strength constant, which we determine by a fit to the ${ }^{3} \mathrm{H}$ binding energy and the $n d$ doublet scattering length ${ }^{2 a_{n d}}$ [35]. The $2 \pi$-exchange $3 N$ force depends on the $N N \pi \pi$ vertex that also occurs in the $2 \pi$-exchange $N N$ force at NNLO. This is one example exhibiting the consistency between $N N$ and $3 N$ forces, which is not guaranteed in the conventional scenario of nuclear forces. In this application we use the low-energy constants related to that $N N \pi \pi$ vertex at NNLO somewhat modified from the values extracted in the $N N \pi \pi$ system to avoid spurious $N N$ bound states [36]. This choice of the modified constants will be avoided in the near future using a newly developed regularization scheme based on a spectral function representation of the pion loop integrals $[37,38]$. Furthermore, the description of $N N$ phase shifts is improved significantly by adding N3LO forces [39]. At this order, a whole host of parameter free $3 \mathrm{~N}$ forces will appear with new spin structures. Also, higher orders in the $p / m$ expansion can be systematically taken into account, which will allow us to investigate relativistic corrections [39]. In view of this work under progress, the results presented here have to be considered only as a very first step. But they serve 
as a quite promising orientation about results gained in that effective-field theory approach to nuclear forces.

\section{B. The $3 N+\Delta$ Hilbert Space Approach}

An alternative theoretical description is given in the framework employed in Ref. [40]. The dynamics is based on the charge-dependent (CD) Bonn potential [41] and its coupled-channel extension allowing for the single excitation of a nucleon to a $\Delta$ isobar [42]. This extension called CD-Bonn + $\Delta$ is a high-quality fit to $N N$ data as CD-Bonn is. The $\Delta$ isobar excitations are believed to be the most important origin of the $3 N$ forces in the $3 N$ Hilbert space approach and are the important origin of the Fujita-Miyazawa type [43] and of the Illinois ring type [44] $3 N F$ s. The contributions are based on all meson exchanges (i.e., $\pi, \rho, \sigma$, and $\omega$ exchanges), contained in the coupled-channel potential; the $\Delta$ propagation is retarded. The arising effective $3 N F$ is much richer with respect to $\Delta$ excitation and also has shorter range components than standard irreducible two-pion exchange $3 N F$. Furthermore, all its components are dynamically consistent with each other and with the effective two-nucleon force. The considered experimental energies stay below pion production threshold; thus, the $\Delta$ isobar can be taken as a stable baryon of spin and isospin $\frac{3}{2}$ with a real mass of $1232 \mathrm{MeV}$. In addition to the $\Delta$-mediated $3 N F$, an irreducible $3 N F$ covering other physics mechanisms is not used. This is the reason why the coupled-channel potential $\mathrm{CD}$-Bonn $+\Delta$ underbinds tritium with $-8.30 \mathrm{MeV}$ [42], the corresponding value for the purely nucleonic reference potential CD-Bonn being $-8.00 \mathrm{MeV}$; the experimental value is $-8.48 \mathrm{MeV}$.

The AGS three-nucleon scattering equations [45] are solved in momentum space without explicit Coulomb interaction between the two protons; the studied observables are not expected to be affected by the Coulomb interaction in the chosen experimental conditions. The calculations use chargedependent potentials with their hadronic proton-proton and neutron-proton parts. The scattering equations are solved as in Ref. [40], using the Chebyshev expansion of the twobaryon transition matrix as interpolation tool. Otherwise, the solution is exact; it appears well converged with respect to angular momentum cutoffs, that is, the two-baryon potential is assumed to be zero for two-baryon total angular momentum larger than 5 and all three-particle partial waves up to threebaryon total angular momentum $\frac{31}{2}$ are included.

Purely nucleonic calculations are done for all modern high-quality potentials; they yield qualitatively similar results and agree well with the results of the model outlined in Sec. II A. However, high-quality coupled-channel extensions have not been constructed yet for the Nijmegen I and II and for the AV18 potentials. Thus, only results for CD-Bonn and its coupled-channel extension CD-Bonn $+\Delta$ will be discussed in this article.

\section{EXPERIMENTAL SETUP}

All experiments presented in this article were performed at KVI. The protons were obtained from the KVI polarized ion-source (POLIS) [46], when polarized ions were used, or the CUSP source, in case the unpolarized beams were utilized. The protons were accelerated in the superconducting cyclotron AGOR to the requested kinetic energy. Because the lower energy limit for extracted polarized protons is $120-\mathrm{MeV}$, protons with a kinetic beam energy of $108 \mathrm{MeV}$ were obtained by degrading $120-\mathrm{MeV}$ protons. When polarized protons were used, the degree of beam-polarization was measured using the KVI In-Beam-Polarimeter (IBP) [47], which is located halfway up the high-energy beam-line. The measurements of the analyzing powers and the differential cross sections of the reaction ${ }^{2} \mathrm{H}(\vec{p}, d p)$ were done using the KVI Big-Bite Spectrometer (BBS) [48] in combination with the EuroSuperNova focal-plane detection system (ESN) [49]. In the following, a brief outline of the measurement and analysis techniques will be given. Further details can be found in Ref. [50].

\section{A. Polarization Measurements}

During the measurements of the analyzing powers and the differential cross sections with polarized protons, the polarization was measured continuously with the IBP using the reaction $\mathrm{H}(\vec{p}, p p)$. General information on the setup and the measurement procedure using the IBP can be found in Ref. [47]. In the following, a short overview of the issues related directly to the measurements done in this work will be given.

Measurements with a $10 \mathrm{mg} / \mathrm{cm}^{2}$ thick $\mathrm{C}_{2} \mathrm{H}_{4}$ target of IBP do not affect the beam current. Furthermore, the introduction of beam-halo because of the presence of the IBP target did not influence the measurements of the differential cross section or the analyzing power with BBS located about $15 \mathrm{~m}$ downstream of IBP and after two bending magnets. Therefore, the polarization could be measured continuously so that the exact polarization value at each measured data point of the reaction ${ }^{2} \mathrm{H}(\vec{p}, d p)$ is known.

The polarization degree $p^{i}$ of the protons obtained from measurements in a plane with azimuthal angle $\phi^{i}$ (from the scattering plane; $i$ is the index representing one of the IBP planes at $0^{\circ}, 45^{\circ}, 90^{\circ}$, and $135^{\circ}$ ) was determined from the measurements of the IBP using the following:

$$
p^{i}=\frac{1}{A_{y}^{p} \cos \phi^{i}} \frac{L^{i}-R^{i}}{L^{i}+R^{i}},
$$

where $L^{i}$ and $R^{i}$ symbolically represent the number of counts on opposite sides of the beam axis in the plane with the azimuthal angle $\phi^{i}$, assuming that there is no asymmetry in the system. For the $0^{\circ}$ plane, $L$ corresponds to $\phi=0^{\circ}$ and $R$ to $\phi=180^{\circ}$. For vector-polarized ions, only the instrumental asymmetry can be measured in the plane with $\phi^{i}=90^{\circ}$ and $\cos \phi^{i}$ should be omitted from Eq. (1) or any of the following equations. The analyzing power $A_{y}^{p}$ of the elastic proton-proton scattering reaction can be obtained with high precision from the existing fits to the world data such as that from the Nijmegen database, PWA93 [51]. The uncertainty in the prediction of the value of the analyzing power from the Nijmegen database is $<2 \%$ [52]. 
When determining the polarization, the instrumental asymmetry of the IBP has to be taken into account. This is done by measuring the counts $L_{0}^{i}$ and $R_{0}^{i}$ of each plane $i$ with an unpolarized beam. The unpolarized beam is obtained by turning the hexapole magnetic field and the transition units of POLIS off. The number of events measured with a polarized proton beam were then normalized to these hexapole-off runs, and the polarization degree of the protons was calculated for each plane from [53] the following:

$$
\begin{aligned}
p^{i} & =\frac{1}{A_{y}^{p} \cos \phi^{i}} \frac{Y_{L}^{i}-Y_{R}^{i}}{Y_{L}^{i}+Y_{R}^{i}}, \\
\text { with } Y_{L}^{i} & =\frac{L^{i}}{L_{0}^{i}} \text { and } Y_{R}^{i}=\frac{R^{i}}{R_{0}^{i}} .
\end{aligned}
$$

To obtain the polarization degree of the protons from the results of the four planes of the IBP, a function

$$
P(\phi)=A \cos (\phi+\alpha),
$$

with the free parameters $A$ and $\alpha$ was fitted to the polarizations obtained from the four planes; $\alpha$ is the angle between the polarization vector and the normal to the horizontal plane. From this function, a normal and a sideways component of the polarization were calculated as follows:

$$
\begin{aligned}
& P_{n}=A \cos \alpha, \\
& P_{s}=A \sin \alpha .
\end{aligned}
$$

Ideally, the polarization vector of the incoming protons should be normal to the horizontal plane and, therefore, $\alpha=0$. During the experiments, the sideways component turned out to be in general $P_{s}<1 \%$ and could thus be neglected. In Fig. 1, the normal components of the up and down polarizations, corresponding to the strong and weak magnetic fields of POLIS, are plotted as a function of time (run number) for $150-\mathrm{MeV}$ incident energy. Each couple of data points generally corresponds to a measurement of the proton-deuteron analyzing power at a certain scattering angle. For some data points, several measurements of the polarization were made. The uncertainties shown in these pictures contain only the statistical ones which are because of the quantities $L$ and $R$. The horizontal lines show the weighted averages of the polarizations when the polarization was constant during the measurement (as is the case in Fig. 1) according to the procedure outlined below. This is done to increase the statistical accuracy of the polarization measurements. When the polarization of the beam changed substantially and for a long period during the run, this averaging was then done for a particular time window in which the polarization was approximately constant.

When calculating the weighted average of the data points as follows:

$$
\begin{aligned}
P_{n}(\text { Energy }) & =\frac{1}{\sum_{k=1}^{N}\left(\frac{1}{\Delta p_{n}^{k}}\right)^{2}} \sum_{k=1}^{N} \frac{P_{n}^{k}}{\left(\Delta p_{n}^{k}\right)^{2}} \\
\chi^{2} & =\frac{1}{N-1} \sum_{k=1}^{N}\left(\frac{P_{n}-P_{n}^{k}}{\Delta p_{n}^{k}}\right)^{2},
\end{aligned}
$$

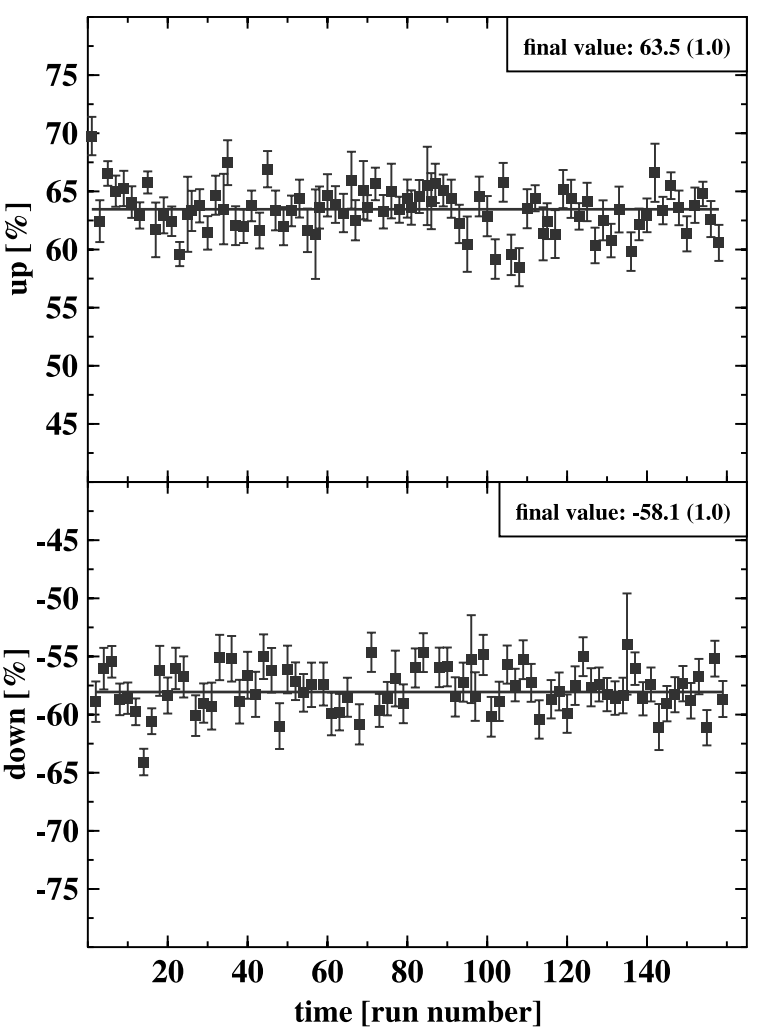

FIG. 1. Polarization measured at $150 \mathrm{MeV}$ incident-beam energy using the IBP. The total time-span for the measurement is about $20 \mathrm{~h}$.

where $P_{n}^{k}$ corresponds to the individual measurements, the statistical error that is because of the hexapole-off run should not be propagated in the individual uncertainties $\Delta p_{n}^{k}$. Otherwise, the uncertainty of the hexapole-off measurement would contribute to every data point and to the $\chi^{2}$ as often as there are data points, leading to a wrong mean and a too small $\chi^{2}$.

If the polarization turned out to be constant within the statistical fluctuations for one energy over a certain time window, with $\chi^{2} \approx 1$, the polarization is determined during this time with higher accuracy. In such a case, all the counts for $L$ and $R$ for each plane can be added and the polarization degree for the different magnetic fields can be determined with a higher accuracy from these sums. These sums have to be normalized to the hexapole-off runs. In this way, also the statistical uncertainties because of $L_{0}$ and $R_{0}$ can be taken into account in the correct way. The polarization degrees of the protons were then determined by calculating the following:

$$
P_{i}=\frac{1}{A_{y}^{p} \cos \phi^{i}} \frac{\sum_{j} L_{j}^{i} / L_{0}^{i}-\sum_{j} R_{j}^{i} / R_{0}^{i}}{\sum_{j} L_{j}^{i} / L_{0}^{i}+\sum_{j} R_{j}^{i} / R_{0}^{i}},
$$

where $i$ indexes the planes at $0^{\circ}, 45^{\circ}, 90^{\circ}$, and $135^{\circ}$ and $\sum_{j}$ is the sum over all counted events. Again, the normal and sideways components of the polarizations were obtained from a fit of the $P_{i}$, as has been described before. For the cases where the $\chi^{2}$ was somewhat larger than 1, a larger error value was added to the data points so that the $\chi^{2}$ is reduced to 1 . This extra error is taken as systematic error of the polarization measurement. The degree of polarization 


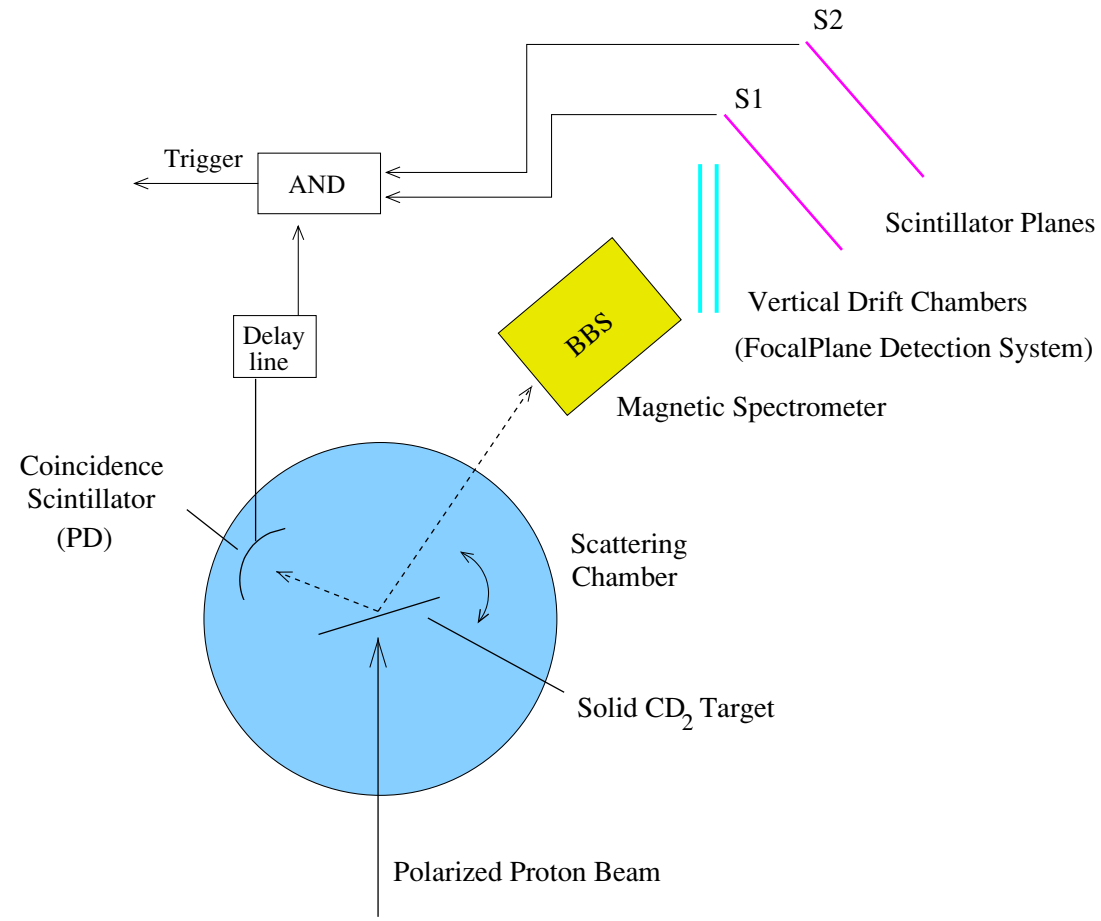

FIG. 2. (Color online) Scheme of the setup of scattering chamber, BBS, ESN, and the coincidence detector (PD). Of the ESN detection system, only those parts that were used during the experiment are shown.

was typically $\approx 60 \%$ for both up and down polarizations. Exceptions are at $190-\mathrm{MeV}$ incident-beam energy, where the polarization was at $\approx 50 \%$ during part of the measurements. During the measurements of $A_{y}$ at $120-\mathrm{MeV}$ incident-beam energy from Ref. [21], the transition unit providing protons with polarization up was not operational and the measurements were performed using down- and off-polarization. It should be noted that there exists an offset of $\approx 8 \%$ in the off-polarization.

The uncertainty in the polarizations turned out to be in general $\Delta p / p<3 \%$. To account for all possible systematic uncertainties, it was set to $\Delta p / p=3 \%$. This $3 \%$ systematic uncertainty also includes the uncertainty of $<2 \%$ in the prediction from the Nijmegen potential. For few data points at $190-\mathrm{MeV}$ incident-beam energy, $\Delta p / p \leqslant 4 \%$.

\section{B. The BBS/ESN Setup}

For the measurement of the ${ }^{2} \mathrm{H}(\vec{p}, d p)$ reaction, two detection systems were used. The magnetic BBS [48] was used together with the ESN [49] for the detection of one of the outgoing particles while a plastic scintillator, placed inside the scattering chamber, detected the coincident particle emerging from the reaction. Aside from the first measurements of the analyzing powers at energies of 120,135, 150, and $170 \mathrm{MeV}$ [21], in which a time coincidence between the outgoing particles was required everywhere, this coincidence detector was used for some runs for cross check with singles measurements and also for a few angles where more was needed to identify the particles in the event than the detection of one of the particles. A schematic drawing of the setup of the experiment is shown in Fig. 2.

Because the BBS can be rotated between $-10^{\circ}$ and $55^{\circ}$, the scattering chamber is constructed in a way to be able to follow these rotations. The fixed beam line is, therefore, connected to the scattering chamber via a sliding seal. The lid holding the coincident detector can be rotated independently of the rest of the scattering chamber.

Inside the scattering chamber, several targets were mounted on a target ladder. This target ladder can hold up to five targets. Two of the target positions were occupied by a $\mathrm{ZnS}$ target, used for focusing the beam, and an empty-frame target for monitoring the beam halo. The other target positions were occupied by $\mathrm{C}_{2} \mathrm{D}_{4}$ targets. Target thicknesses of 10,20 , and $50 \mathrm{mg} / \mathrm{cm}^{2}$ were used for various angles and energies depending on the count rate. For the measurements of $A_{y}$ at 120, 135, 150 , and $170 \mathrm{MeV}$ [21], pure $\mathrm{C}_{2} \mathrm{D}_{4}$ targets were used. For the measurements of cross sections at all energies and analyzing powers at 108 and $190 \mathrm{MeV}$, and the second measurements of analyzing powers at 120 and $150 \mathrm{MeV}$ (for cross check with the earlier results of [21]), a mixture of $\mathrm{C}_{2} \mathrm{H}_{4}$ and $\mathrm{C}_{2} \mathrm{D}_{4}$ with the atomic ratio of $\mathrm{D}: \mathrm{H}$ of $87 \%: 13 \%$ was used for the targets. The hydrogen content was used in the $\mathrm{H}(p, p)$ reaction to determine the target thickness by comparing the experimental cross sections with the well-known cross sections of Ref. [51].

These targets were used for the measurement of the ${ }^{2} \mathrm{H}(\vec{p}, d p)$ reaction. The target ladder could be rotated to allow for detection of low-energy particles emerging from the scattering process and crossing the target.

At $\theta_{\text {lab }}=5^{\circ}$, a Faraday cup inside the BBS was used. The position of this Faraday cup had to be adjusted for the magnetic setting of the dipole. At this angle, a measurement of the reaction $\mathrm{H}(p, p)$ for the determination of the target thickness was not sensible as a change in the magnetic rigidity would result in the change of position of the Faraday cup. For laboratory scattering angles $8.5^{\circ} \leqslant \theta_{\text {lab }} \leqslant 14^{\circ}$, a Faraday cup was used which was fixed in the BBS between the quadrupole magnets $Q_{1}$ and $Q_{2}$. For laboratory scattering angles larger 


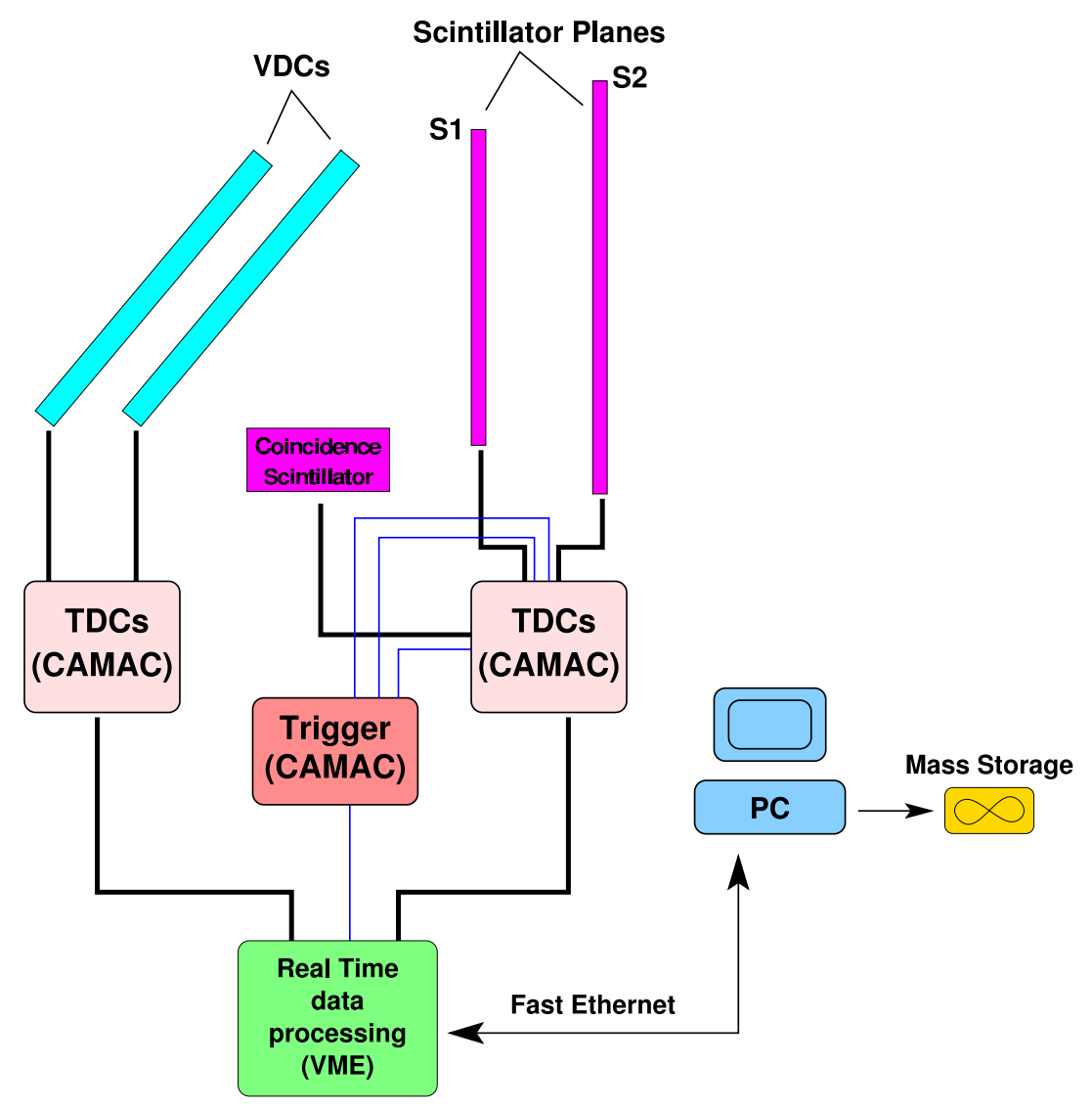

FIG. 3. (Color online) Diagram of the electronics readout system of the BBS/ESN focalplane detection system and the PD coincidence detector. than $14^{\circ}$, a Faraday cup was installed inside the scattering chamber to stop the beam and to measure the beam current. This Faraday cup consists of a copper block containing a heavy metal alloy as the actual beam stopper. With the second and the third Faraday cups, measurements of $\mathrm{H}(p, p)$ reaction were performed for selected angles to obtain the absolute normalization of the measurements.

During the experiment, measurements were made with the BBS/ESN detection system at angles between $5^{\circ}$ and $53^{\circ}$. At angles above $11^{\circ}$, measurements were made with the dipole magnetic field of the BBS adjusted alternately to select the outgoing proton or the outgoing deuteron. At angles below $11^{\circ}$, measurements were done only with the dipole field adjusted for selecting the deuteron. The reason was that the proton detection with the BBS at small angles in coincidence with the outgoing deuteron was not possible, because the kinetic energy of the corresponding deuteron is too low to traverse the target and reach the coincidence detector. For singles measurement of protons with the BBS at these angles, the following problem arose. Protons from the ${ }^{12} \mathrm{C}\left(p, p^{\prime}\right){ }^{12} \mathrm{C}$ reaction entering the BBS have a similar momentum as protons emerging from the ${ }^{2} \mathrm{H}(\vec{p}, d p)$ reaction because of very small difference in the recoil energy of the nuclei at these small angles. These processes could, therefore, not be distinguished at angles below $11^{\circ}$ resulting in a large background. Therefore, no measurements with protons entering the BBS were done for these angles.

The electronics readout of the BBS/ESN detection system, shown in Fig. 3, is identical to the electronics setup used by the ESN collaboration [49] for those parts of the detectors, that were used during this experiment. For the signal processing, the KVI data-acquisition software $c d a q$ [54] was used. The event buffer was sent via a network cable to a UNIX computer. There, the data stream was written to tape. Part of the data stream was used for the online analysis. The number of various triggers were also written to CAMAC scaler units (LeCroy 4434). In one of the scaler channels, the information about the beam polarization was stored. Further, the collected charge from the Faraday cup, the number of the acquired events, and the global time were stored in the scaler units. The scaler units were read out about every $10 \mathrm{~s}$ independent of the real event triggers.

The event rates varied typically between about $100 \mathrm{~Hz}$ in the minima of the differential cross sections and several kilohertz at small scattering angles where the differential cross sections are the largest. During the measurements of the differential cross sections, the computer dead times were, in general, kept below $20 \%$ and corrected for accordingly.

\section{ANALYSIS OF THE DATA}

\section{A. Analysis of the Raw Data}

The experiments reported here were performed at various times. The cross sections were obtained either with polarized protons (at 108, 120, 150, and $190 \mathrm{MeV}$ ) or with unpolarized protons (at 135 and $170 \mathrm{MeV}$ ). For the latter energies, measurements were performed in separate runs with polarized protons to determine the analyzing powers. 

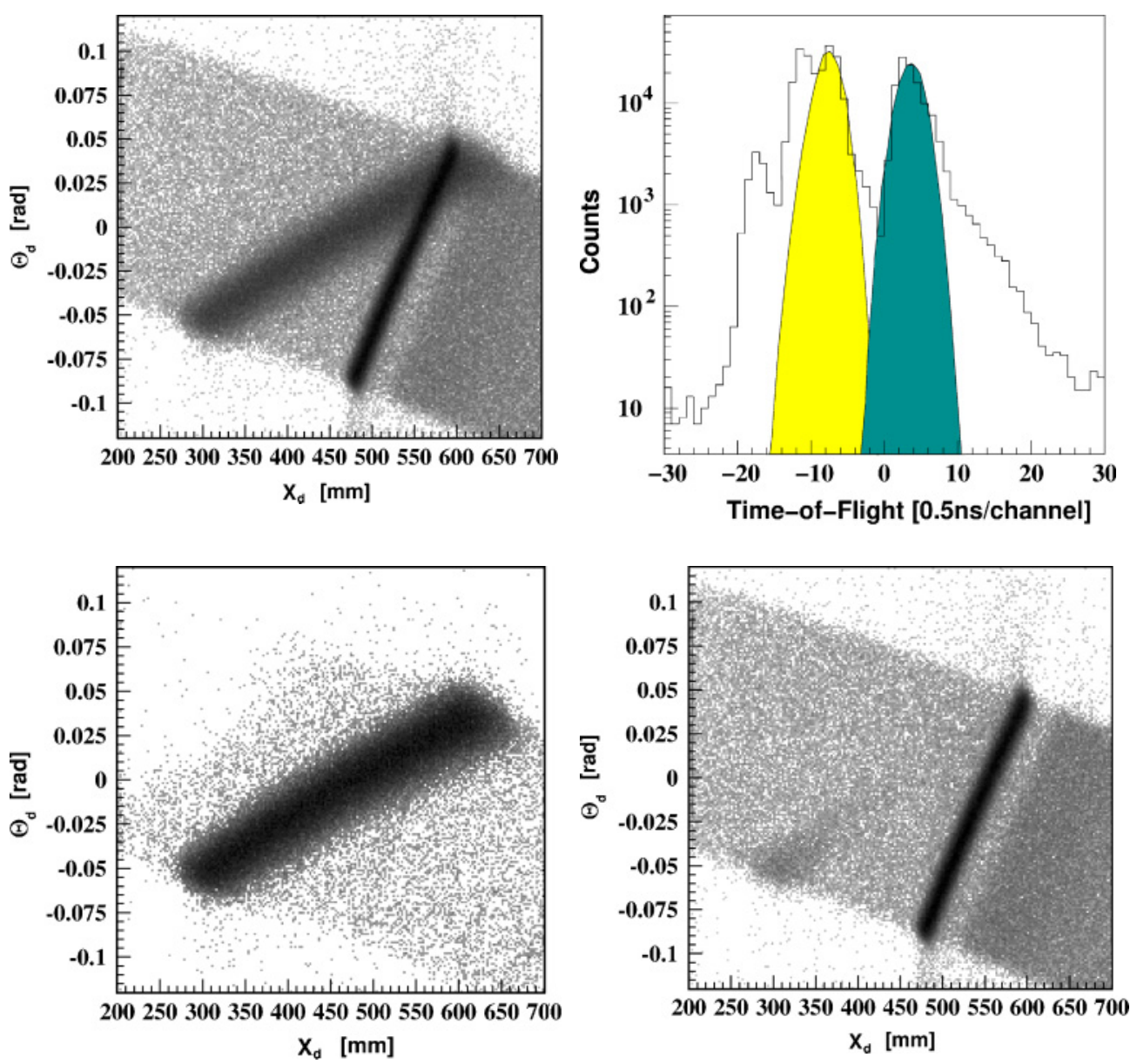

FIG. 4. (Color online) Shown are events at a bombarding energy of $150 \mathrm{MeV}$ for a scattering angle of $50^{\circ}$. At this angular setting, protons and deuterons emerging from the ${ }^{2} \mathrm{H}(\vec{p}, d p)$ reaction are both scattered to the same angle with a similar magnetic rigidity. Furthermore, background stemming from the ${ }^{12} \mathrm{C}\left(p, p^{\prime}\right)^{12} \mathrm{C}$ reaction has been recorded. In the upper-left figure, the two-dimensional spectrum of the incidence angle versus position at the focal plane is shown. The broad dark band represents the scattered deuterons, the narrow dark band shows the protons from the same reaction. In the upper-right figure, the ToF spectrum (see text) for the same setting is shown, with the left (light-shaded) peak corresponding to protons and the right (dark-shaded) peak corresponding to deuterons. In the lower-left figure, the deuteron band has been selected by a cut on ToF and in the lower-right figure the proton band.

In the analysis of the raw data, the trajectories of the particles at the focal plane were constructed from the Time-toDigital Converter (TDC) signals of the vertical drift chambers (VDCs). These trajectories are expressed in the Cartesian coordinates by $X_{d}$ and $Y_{d}$ and the relative scattering angles $\theta_{d}$ and $\phi_{d}$ with respect to the central ray at the focal plane. For further analysis, the $x$ position corrected for kinematical broadening at the focal plane, $X_{f}$, was calculated [55]. From the TDC signals of the two scintillator planes $S 1$ and $S 2$, the time-of-flight (ToF) between the two planes and timeover-threshold (ToT) signal for each scintillator paddle were determined.

Sources of background were protons stemming from the reaction ${ }^{12} \mathrm{C}\left(p, p^{\prime}\right)^{12} \mathrm{C}$ and, at a laboratory scattering angle around $50^{\circ}$, the reaction ${ }^{2} \mathrm{H}(\vec{p}, d p)$ itself. Here, protons and deuterons from this reaction are scattered into the same angular range with a similar magnetic rigidity. Therefore, both particles will be detected simultaneously with the BBS/ESN. In Fig. 4, an example of a two-dimensional spectrum measured at the focal plane is shown where both the outgoing protons and deuterons of the reaction ${ }^{2} \mathrm{H}(\vec{p}, d p)$ are measured with the focal-plane detection system. To reduce the background stemming from either of the two reactions, as is shown in Fig. 4, the ToF between the two scintillator planes SI and $S 2$ was used. Because deuterons and protons with the same momentum-to-charge ratio, and thus the same magnetic rigidity, have different velocities, the ToF between $S 1$ and $S 2$ differs and can be used to separate them. The ToF spectrum, where the two peaks corresponding to deuterons and protons from the reaction ${ }^{2} \mathrm{H}(\vec{p}, d p)$ can be distinguished, is also shown. Both peaks were fitted with a Gaussian distribution to help place the cuts properly. The actual cuts were made such that very few valid events of either reaction were cut away. The two two-dimensional spectra of the focal plane obtained with these cuts in the ToF spectrum are shown at the bottom of Fig. 4.

For other angles, the main background in the focal plane was because of the carbon contained in the polyethylene matrix 

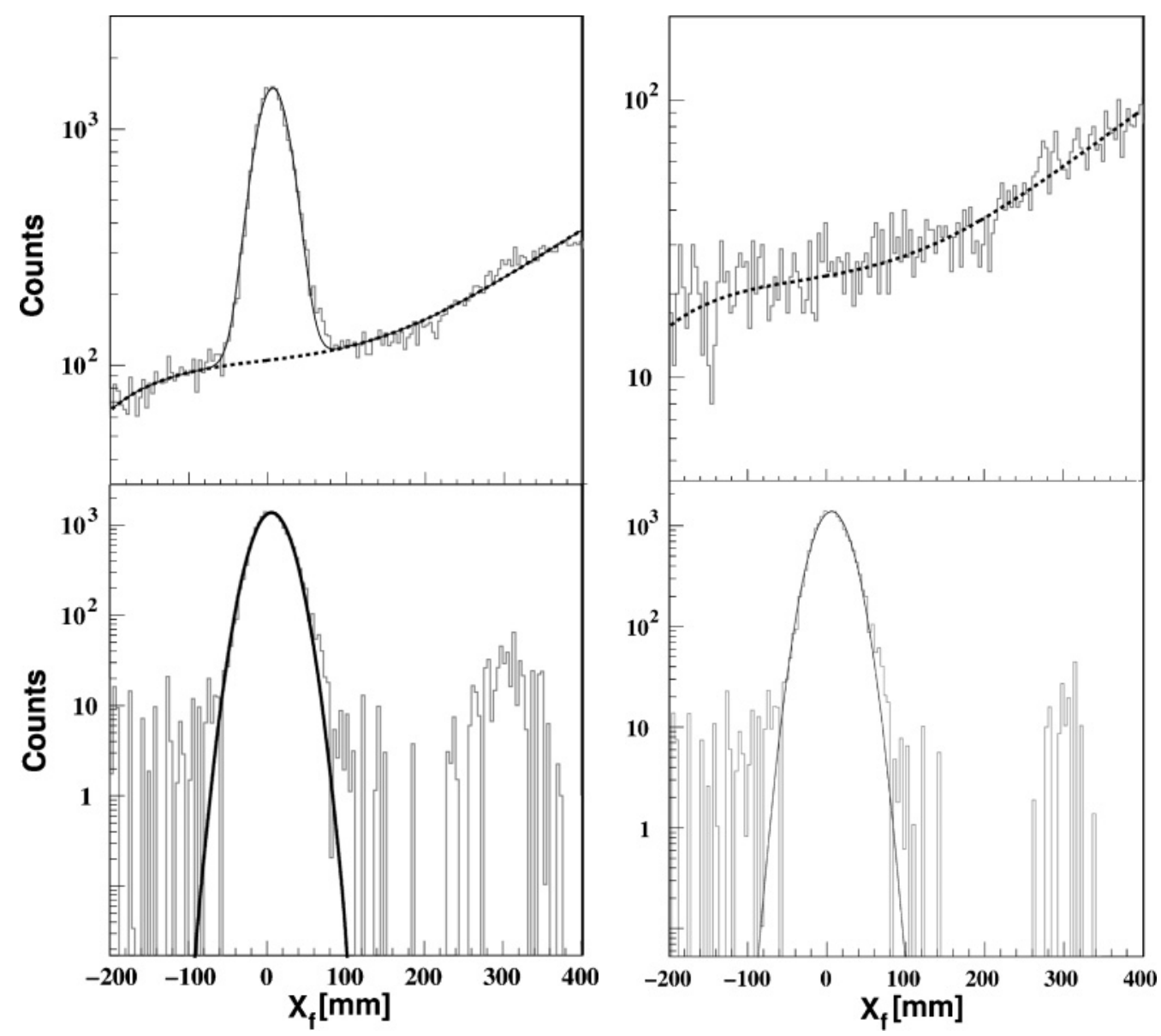

FIG. 5. Background subtraction for spectra of the virtual focal plane. In the upper-left panel, a spectrum of the position $X_{f}$ of deuterons entering the BBS is shown along with the result of the Gaussian+polynomial fit and the result of the polynomial fit on its own. In the lower-left panel, a histogram is shown where the polynomial fit has been subtracted from the spectrum shown in the upper-left panel. In the upper-right panel, a spectrum for the same settings as in the upper-left panel, but taken with a pure carbon target is shown together with a polynomial fit of the spectrum. In the lower-right panel, a histogram is shown where the polynomial fit of the spectrum taken with the carbon target has been subtracted from the histogram shown in the upper-left panel. The agreement between the two lower spectra is good as expected.

of the target. To subtract this background, the focal-plane spectrum $X_{f}$ of all particles was fitted with a polynomial and a Gaussian. The polynomial represents the background and the Gaussian the peak of interest. To check whether the background because of carbon was subtracted sufficiently when using a polynomial fit, as described, measurements were done using a pure carbon target. The spectra of $X_{f}$ recorded during these measurements were also fitted with a polynomial. The result of that fit was, after correcting for the different luminosity, subtracted from the corresponding spectrum measured with a $\mathrm{C}_{2} \mathrm{D}_{4}$ target. An example is shown in Fig. 5. In the upper left panel, a one-dimensional histogram of the recoil-corrected focal-plane position is presented. This histogram was recorded for a setting at $170-\mathrm{MeV}$ incidentbeam energy with the deuteron emerging from the reaction ${ }^{2} \mathrm{H}(\vec{p}, d p)$ entering the $\mathrm{BBS}$ at $38^{\circ}$. In this histogram, the result of a fit using a sum of a Gaussian and a polynomial and the result for the polynomial part of the fit are shown. In the histogram placed in the lower-left panel of Fig. 5, the polynomial has been subtracted from the measured spectrum shown in the upper-left panel. In the upper-right figure, a histogram of a measurement for the same BBS settings but using a carbon target is presented. This histogram was fitted with a third-order polynomial. In the lower-right panel, this polynomial was subtracted, after a proper normalization accounting for the luminosity, from the histogram in the upper-left figure. The difference between the two histograms in the lower panels in Fig. 5 is negligible, showing that the method of fitting as shown in the upper-left panel is indeed sufficient to subtract the carbon background.

\section{B. Correction Factors}

The target thickness and the beam intensity were calibrated by using elastic proton-proton scattering. In these measurements, both protons were detected where the angles and energies of the outgoing particles allowed this. When this was not possible, only one of the outgoing particles was detected. 


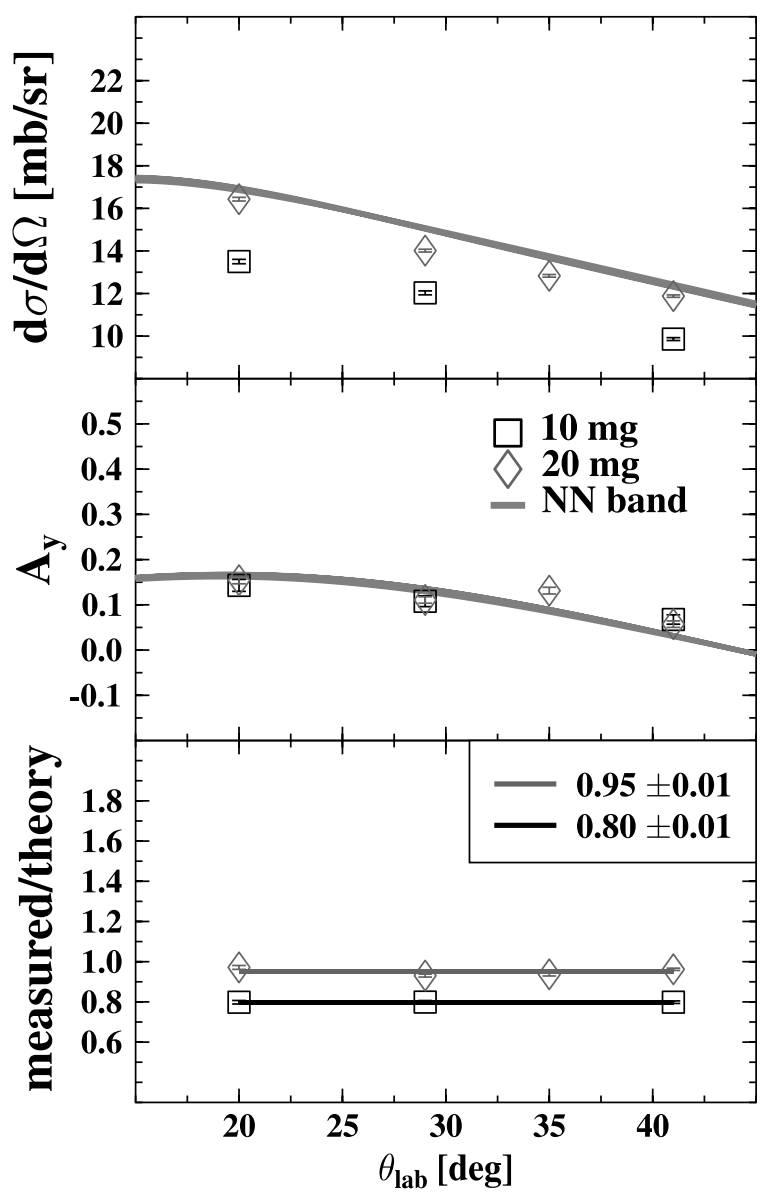

FIG. 6. Results of the measurements of the proton-proton differential cross section and analyzing power at $120 \mathrm{MeV}$ for two different target thicknesses. Note that to obtain the cross sections shown in the upper panel, the nominal values of the target thickness were used leading to different normalizations for different target thicknesses. The weighted averages of the normalization factors for the cross sections obtained for different target thicknesses are shown as bands in the lowest panel.

The analysis showed that the results of singles measurements after a simple background subtraction such as shown in Fig. 5 agrees well with the results obtained in the coincidence measurements. To be consistent in the analysis, only the singles results were used in the analysis. Because the observables of $N N$ scattering can be calculated rather precisely with modern $N N$ potentials, a precise overall normalization factor can be obtained. Measurements of elastic proton-proton scattering were done at several angular settings for all beam energies during the cross-section measurements. In the cases, where polarized protons were used, the comparison of the measured proton-proton analyzing power with the results obtained from the calculation was used as a cross check of the polarization value obtained by IBP. The agreement between these two separate measurements is very satisfactory.

The analysis of the proton-proton scattering data was done in the same way and with the same analysis software as in the analysis of the proton-deuteron scattering data described before. In Fig. 6, a comparison between the measured and the calculated differential cross sections and analyzing powers and the ratio between measurements and calculations for $120-\mathrm{MeV}$ incident-beam energy are shown as an example of one of the six different energies. Note that the experimental cross sections plotted in this figure are obtained by using the nominal values of the target thickness. This could, then, lead to different normalization factors for different target thicknesses. The theoretical calculation of the analyzing power and the differential cross section is shown as a band, obtained from the potentials Nijmegen I, Nijmegen II, [4] and Reid93, and the partial-wave analysis, PWA93 [51]. For further analysis, an average of the results of these four potentials was used, resulting also in a theoretical uncertainty. This error was propagated in the uncertainty of the normalization factors. The normalization factors so obtained generally varied between 0.75 to 1.2 for various target thicknesses and different energies.

Another factor, that should be taken into account is radiation damage to the target. In the case of $\mathrm{C}_{2} \mathrm{H}_{4}-\mathrm{C}_{2} \mathrm{D}_{4}$ targets, radiation damage would induce a breaking of the $\mathrm{C}-\mathrm{C}$ bonding, leading to a loss of hydrogen and deuterium. Also, it is known that the radiation damage depends on the manufacturing process of the target. Nevertheless, some estimate can be made based on known numbers. Because to a good approximation, the effect should be the same for hydrogen and deuterium, the ratio between both contents should not change significantly and the effect on the normalized differential cross section should be small. This assumption was confirmed by massspectroscopic measurements of the ratio that were done after the experiments had been performed [56,57]. These measurements showed that the $\mathrm{C}_{2} \mathrm{D}_{4}: \mathrm{C}_{2} \mathrm{H}_{4}$ ratios in irradiated and nonirradiated regions of the target did not differ. Using an estimation of $\approx 1 \%$ loss of deuterium per $10^{7}$ Gy radiation dose [58], an estimation for the possible radiation damage can be made. The average energy deposit for an incident-beam energy of $108 \mathrm{MeV}$ is $\approx 13 \mathrm{MeV} /\left(\right.$ proton $\cdot \mathrm{g} / \mathrm{cm}^{2}$ ). For a beam area of roughly $5 \mathrm{~mm}^{2}$, a current of $1 \mathrm{nA}$ and a radiation time of $24 \mathrm{~h} \approx 10^{5} \mathrm{~s}$, the deposited radiation would be $\approx 10^{7} \mathrm{~Gy}$, resulting in a decrease of the hydrogen and deuterium content of $\approx 1 \%$. This estimation is only true for the lowest beam energy used in this work; at higher bombarding energies, the deposited energy would be even less. Furthermore, the total systematic uncertainties of the differential cross sections, which will be discussed later (Sec. IVD) are about 5\%; a $1 \%$ effect could, therefore, not be observed. As can be seen in Fig. 6, the normalization factors obtained from elastic proton-proton scattering measured at different times, are rather constant, leading to the conclusion that radiation damage is negligible for the measurements done in this work.

Apart from global corrections, such as the correction for the target thickness and the beam current, which could be accounted for by using the elastic proton-proton differential cross section, efficiencies of the detection system depending on the particle-type of the ejectile had to be corrected for separately. This concerned mainly the efficiency of the VDC detection and the VDC reconstruction with respect to protons and deuterons. 
The efficiency of one plane of the VDCs, $\epsilon_{i}(i=1 \ldots 4)$, was determined as follows:

$$
\epsilon_{i}=\frac{\sum \text { one hit in all four planes }}{\sum \text { one hit in three planes }} .
$$

The total efficiency of the VDC was calculated from the product of the efficiencies of all four planes,

$$
\epsilon_{\mathrm{VDC}}=\prod_{i=1}^{4} \epsilon_{i} .
$$

This efficiency contained the convolution of the detection efficiency and the efficiency of the reconstruction algorithm used to determine the interception point in the plane from the drift times. The efficiency depends on the particle type and the kinetic energy of the particle but is typically larger than $90 \%$.

\section{Physical observables}

In this section, it is briefly outlined how the physical observables are obtained. When the events for a data point were selected according to the cuts described in Sec. IV A and corrected for dead time, the differential cross section was calculated from the number of counts, knowing the number of incoming particles, the target thickness, the solid angle, and the inefficiencies of the detection system. This same procedure was used when the beam was polarized. In this case, the cross section was obtained for each spin state separately.

The relation between the spin-dependent cross section $d \sigma^{s}$ and spin-averaged cross section $d \sigma^{0}$ is as follows:

$$
d \sigma^{s}=d \sigma^{0}\left(1+p^{s} \cdot A_{y} \cdot \cos \phi\right)
$$

for a vector polarization $p^{s}$ and vector analyzing power $A_{y} . \phi$ is the angle between the direction of polarization and the normal to the scattering plane in the laboratory frame of reference. The definition in the literature [59] is that for an incoming proton with spin up, $\phi=0^{\circ}$ corresponds to the scattering of the outgoing proton to the left and $\phi=180^{\circ}$ to the right. For a setting, where BBS selects the outgoing deuterons and the outgoing protons are measured by the coincidence scintillator, the relation between $d \sigma^{s}=d \sigma^{\uparrow}$ and $d \sigma^{0}$ would be as follows:

$$
d \sigma^{\uparrow}=d \sigma^{0}\left(1+p^{\uparrow} \cdot A_{y}\right)
$$

for an incoming proton with spin up. If the incoming proton had spin down, the angle $\phi$ would be shifted by $180^{\circ}$. For the case mentioned here, the scattered proton would see the coincidence scintillator at an angle $\phi=180^{\circ}$ and the relation between $d \sigma^{s}=d \sigma^{\downarrow}$ and $d \sigma^{0}$ would then be as follows:

$$
d \sigma^{\downarrow}=d \sigma^{0}\left(1-p^{\downarrow} \cdot A_{y}\right) .
$$

Because for the measurement with BBS, the acceptance of $\mathrm{BBS}$ at the angles measured is sufficiently small and only the possibilities for $\phi=0^{\circ}$ and $\phi=180^{\circ}$ exist, the minus sign in Eq. (11) could also be absorbed in the polarization, assigning a negative polarization value to the down polarization. In this case, Eqs. (10) and (11) look alike. In all these cases, the polarization degree of freedom is obtained from an IBP measurement as outlined in Sec. III A.

From the two cross sections $d \sigma^{\uparrow}$ and $d \sigma^{\downarrow}$ with the polarization $p^{\uparrow}$ and $p^{\downarrow}$, where $p^{\downarrow}<0$, the analyzing power can be calculated,

$$
A_{y}=\frac{d \sigma^{\uparrow}-d \sigma^{\downarrow}}{p^{\uparrow} d \sigma^{\downarrow}-p^{\downarrow} d \sigma^{\uparrow}} .
$$

Equation (12) holds for any polarization degree, provided that care is taken with the sign of the polarization.

For settings, where the outgoing protons are selected by BBS and the outgoing deuterons are measured with the coincidence scintillator, care has to be taken. For an incoming beam with polarization up, the outgoing protons are scattered to the right (i.e., $\phi=180^{\circ}$ ) and the vector analyzing power calculated from Eq. (12) has to multiplied by -1 .

Once the vector analyzing power has been calculated, Eq. (10) or (11) may be used to calculate the spin-averaged differential cross section $d \sigma^{0}$,

$$
\begin{aligned}
d \sigma^{0} & =\frac{d \sigma^{s}}{1+p^{s} A_{y}} \\
& =\frac{p^{\uparrow} d \sigma^{\downarrow}-p^{\downarrow} d \sigma^{\uparrow}}{p^{\uparrow}-p^{\downarrow}},
\end{aligned}
$$

where the polarization $p^{s}$ can be larger or smaller than zero. Even though the efficiencies are included in the calculation of the cross section, they may be bypassed if the interest lies only in the analyzing powers. These depend only on the ratio of the cross sections and the efficiencies cancel each other. This assumes, of course, that the efficiencies are independent of the spin as was the case for the measurements done in this work.

\section{Errors}

In this section, an overview of all sources of errors will be given for the cross sections and the analyzing powers. There are two classes of uncertainties that should be discussed. The first one deals with the statistical uncertainty of the measurements that is generally very small for both the cross sections and the analyzing powers (see the tables in the appendix).

The second class is the systematic uncertainty that should be treated separately for the two measured observables. In the analysis of the analyzing powers, many sources of systematic uncertainties do not enter in the final number because one is dealing with ratios of cross sections. Most importantly, the luminosity of the experiment is not an issue in these measurements except for the dead-time corrections that have been taken into account properly and are also a second-order correction on the error. The main uncertainty in the analyzing power comes from the determination of the polarization of the beam. This error contributes to about 3\% [47].

The cross section measurements were done either with POLIS or the CUSP source as was discussed in Sec. III. When the polarized source is used, the uncertainty in the polarization enters in obtaining the cross sections. The determination of 
the luminosity also contributes to the systematic uncertainty of the cross sections (these are listed apart in the data tables in the appendix). The luminosity of the measurements were obtained by elastic proton-proton scattering measurements at a few angles during the experiments as was described in Sec. IV B. It is quite conceivable that the beam envelope on the target has moved on the target thereby going through slightly different thicknesses. The fact that the target thickness varies slightly over its surface was confirmed in measurements of the target thickness after the experiments. Also, to obtain cross sections, fits were done to spectra (see Fig. 5) to disentangle the contents of the peak and the background. The systematic uncertainties associated with the background subtraction were estimated to be $\lesssim 3 \%$. To account for all these uncertainties, a fit was made with a polynomial of ninth or eleventh order (depending on the energy) to the measured proton-deuteron cross sections for each energy. It was observed that, even with these high-order polynomials, a $\chi^{2}$ of 1 was not observed, indicating a lack of knowledge of the exact target thicknesses during the measurements at different angles (also at different times) on top of the systematic uncertainties in the background subtraction. Therefore, an error was added to each data point to force the $\chi^{2}$ to go to one. This extra error, which is of the order of $4 \%$, is called the point-to-point systematic uncertainty and varies for different energies. The caption of each table in the appendix includes this number as well.

\section{FINAL RESULTS AND DISCUSSION}

In the following, experimental results obtained for $d \sigma / d \Omega$ and $A_{y}$ for the reaction ${ }^{2} \mathrm{H}(\vec{p}, d p)$ will be presented and compared to calculations including three-nucleon forces $(3 N F)$ from different models and calculations using $N N$ potentials only.

The experimental results for the differential cross section as a function of c.m. scattering angle and bombarding energy are shown in Figs. 7 through 9, where comparisons are made with the calculations within the $3 N$ Hilbert space approach (see Sec. II A). In the first figure, the cross sections are presented as a function of $\theta_{\text {c.m. }}$ angle on a logarithmic scale. To see the differences better at large angles, the region between $90^{\circ}$ and $150^{\circ}$ is shown again on a linear scale in Fig. 8. In Fig. 9, the percentage deviation of theory from experiment is shown for the whole angular range to magnify any systematics. Data points are set to zero with the band of systematic experimental uncertainty shown around them. The results obtained from theoretical calculations are shown as bands for calculations using $N N$ potentials only (black band) and calculations using $N N+\mathrm{TM} 99$ (gray band). Furthermore, results obtained from calculations using AV18+Urbana-IX are presented as solid lines. At $108 \mathrm{MeV}$, also results from calculations based on chiral perturbation theory $(\chi \mathrm{PT})$ are drawn as a dark gray band. The band is the result of using different cutoff values in the calculations. At $135 \mathrm{MeV}$, also data obtained from high-precision measurements of the reaction $\mathrm{H}(\vec{d}, p d)$ at $E_{\text {beam }}=270 \mathrm{MeV}$ by Sakai et al and Sekiguchi et al. $[8,9]$ are shown. At $150 \mathrm{MeV}$, results from measurements done by Postma and Wilson [13] at $146 \mathrm{MeV}$ and by Kuroda et al. [14]

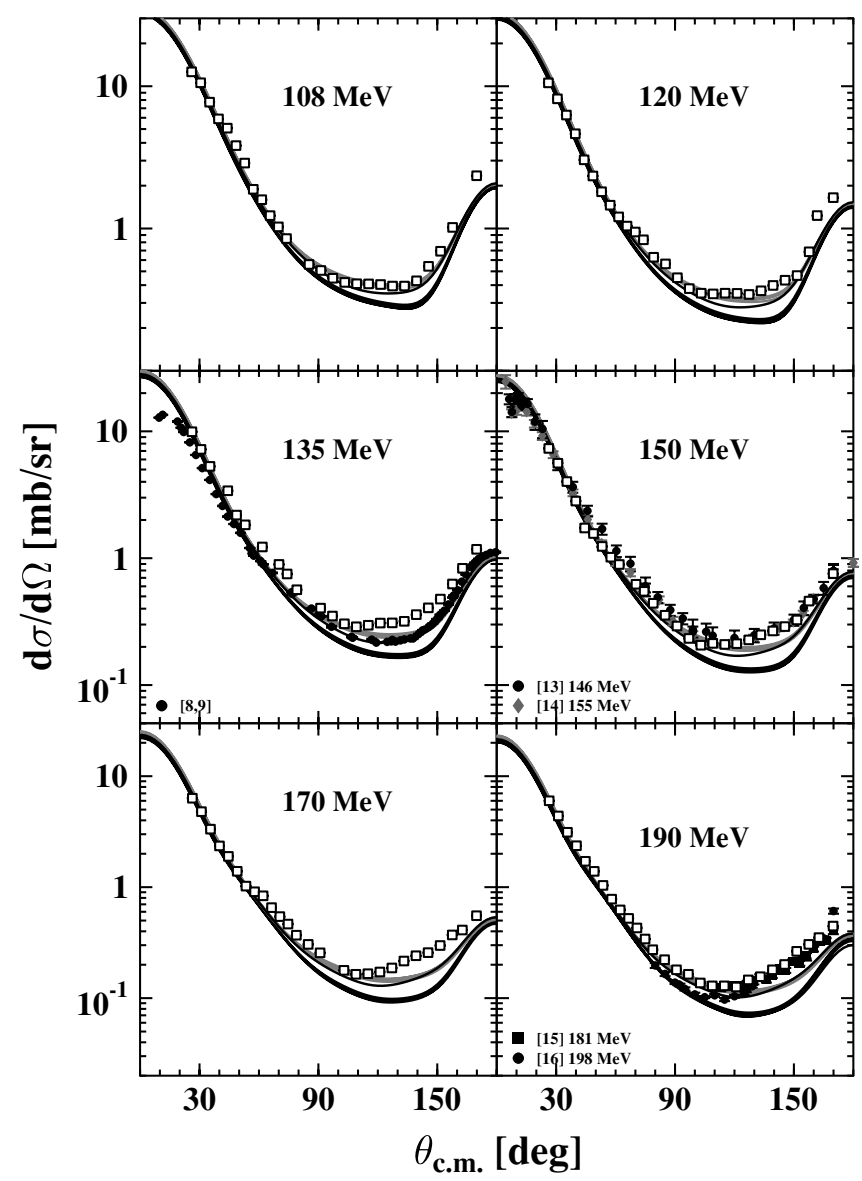

FIG. 7. Differential cross sections for the reaction ${ }^{2} \mathrm{H}(\vec{p}, d p)$ as a function of the scattering angle for different incident-beam energies. Shown are the data set from this work (open squares) and calculations from $N N$ potentials (black band), $N N+$ TM99 (gray band), AV18+Urbana-IX (solid line), and, at $108 \mathrm{MeV}$, from $\chi \mathrm{PT}$ (dark gray band). The solid line overlaps in most places with the gray band. The same holds for the dark gray band at $108 \mathrm{MeV}$. The other data sets shown are denoted in the pictures and explained in the text. The statistical uncertainty is depicted at each data point.

at $155 \mathrm{MeV}$ are included. At $190 \mathrm{MeV}$, data are shown from measurements done by Adelberger and Brown [16] at 198 $\mathrm{MeV}$ and Igo et al. [15] at $181 \mathrm{MeV} /$ nucleon. The data set from Ref. [15] at $217 \mathrm{MeV}$ has been omitted, as the difference in kinetic energy is too large for a meaningful comparison. Also the data set measured with a $185 \mathrm{MeV}$ proton beam [60], which agrees reasonably well with the data presented here, has been left out because it covers a very small angular range. As can be seen, the data obtained in the present work shows a systematic deviation from the data of reference $[8,9]$ of $\approx 25 \%$. Also the shape of the cross sections of the two measurements are different. A small part of the difference can be accounted for by systematic uncertainties of both experiments. Recent cross sections results from IUCF (not absolute cross sections) at this energy seem to agree well in shape with our data [61]. Furthermore, given the uncertainties and incident beam-energy differences in various experiments, the present data agree 


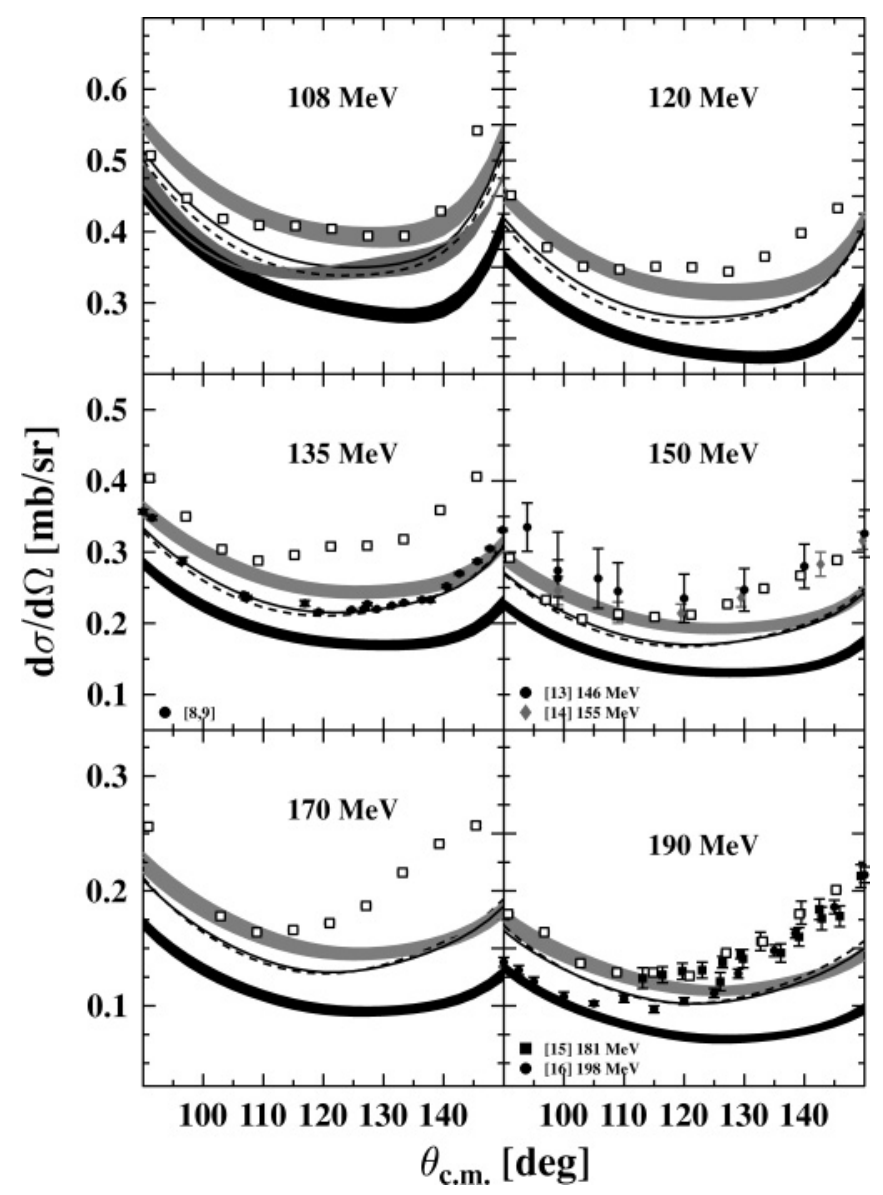

FIG. 8. The differential cross sections for the angular range between $90^{\circ}$ and $150^{\circ}$ on a linear scale as opposed to the logarithmic scale of Fig. 7. For explanation of curves and data, see Fig. 7 with the lower gray band in $108-\mathrm{MeV}$ panel from the $\chi \mathrm{PT}$ calculations. The extra curve shown by dashed line is for CD-Bonn $+\Delta$ of Fig. 10.

rather well with data sets at other energies $[13,14,16]$ with the possible exception of the last backward-angle data points at $181 \mathrm{MeV}$ from Ref. [15]. The shape of the angular distribution of the differential cross section obtained in this work at $190 \mathrm{MeV}$ is also in rather good agreement with data taken earlier at KVI with SALAD [62], which are not shown here.

For the sake of clarity, the comparison of the data obtained in this work with calculations within the $3 \mathrm{~N}+\Delta$ Hilbert space approach of the Hanover group [42] (and Sec. II B) is shown in separate figures (Figs. 10 and 11). Again, the second figure is simply presented to enhance subtle effects. Results are shown from calculations using the purely nucleonic CD-Bonn potential (dashed lines) and its coupled-channel extension CD-Bonn $+\Delta$ (solid lines).

In the angular range between $30^{\circ} \lesssim \theta_{\text {c.m. }} \lesssim 60^{\circ}$, there seems to be small disagreements between the theory and the data, depending on the incident energy. Furthermore, the deviations of the theoretical predictions from our data are slightly larger than the systematic uncertainty of the data. At

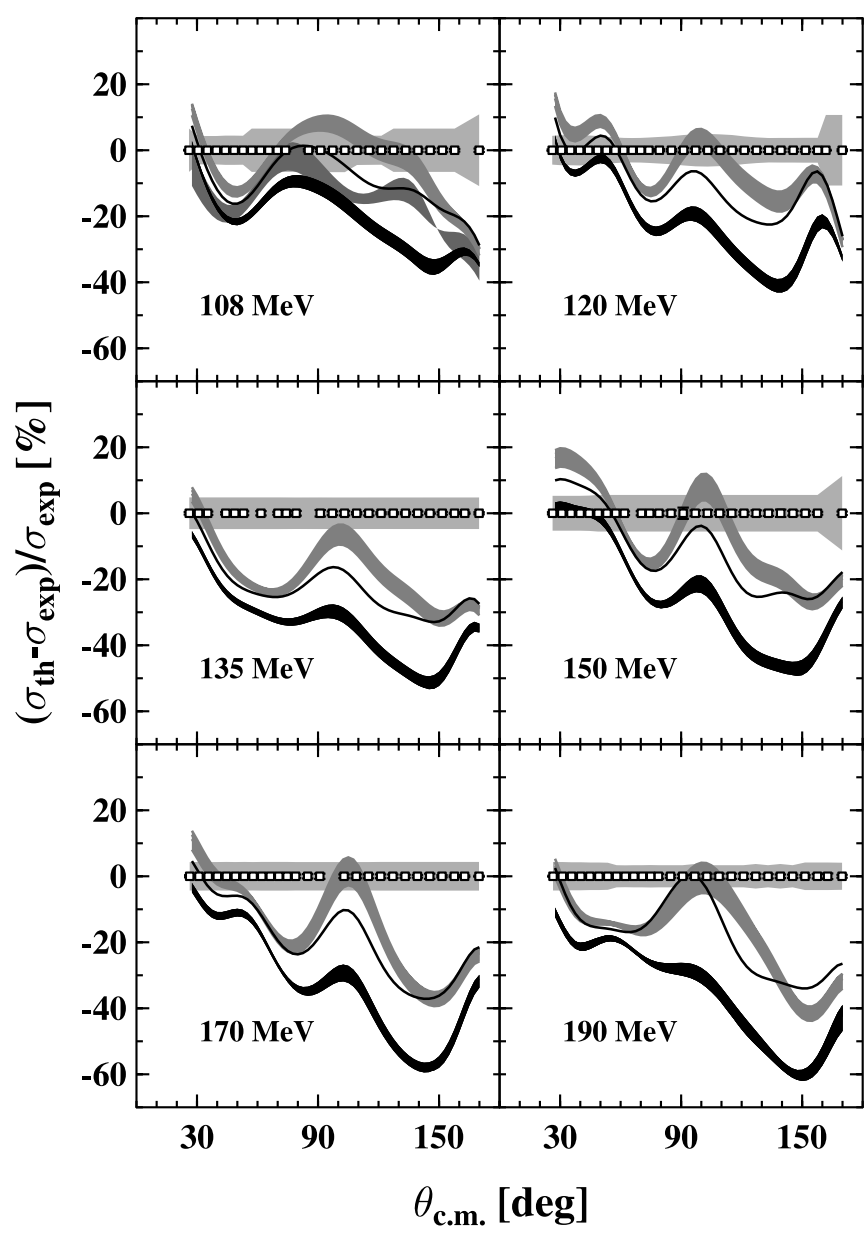

FIG. 9. Shown are the deviations of the different theoretical calculations from the data sets measured in this work for the differential cross section of the reaction ${ }^{2} \mathrm{H}(\vec{p}, d p)$. The meaning of the curves is the same as in Fig. 7. The gray band around zero represents the systematic uncertainty of the measurement. Further information about the presentation is given in the text.

$\theta_{\text {c.m. }} \geq 60^{\circ}$, the $N N$ band and the $N N+$ TM99 band deviate from each other. Calculations using two-nucleon interactions only completely fail to describe the data for the rest of the angular range. The deviation between calculations using only $N N$ potentials and the data is largest around $130^{\circ} \lesssim \theta_{\text {c.m. }} \lesssim 150^{\circ}$. This angular range is part of the region of the minimum and the place, where $3 \mathrm{NF}$ effects would be expected to show up [10].

The use of additional $3 \mathrm{NF}$ or inclusion of explicit Delta's remedies these discrepancies at the lower energies for both model calculations. For $60^{\circ} \lesssim \theta_{\text {c.m. }} \lesssim 150^{\circ}$ and for energies $\lesssim 150 \mathrm{MeV}$, the data set is described rather well by the $N N+3 N$ band. All $3 N F$ used in these calculations, TM99, UrbanaIX, and explicit $\Delta$ excitation, give similar results. Around $\theta_{\text {c.m. }} \approx 70^{\circ}$, a local minimum appears in the difference plots (i.e. Figs. 9 and 11). This minimum is because of a "shoulder" of the differential cross section, which begins at $\theta_{\text {c.m. }} \approx 60^{\circ}$ and is shown by the calculations and, more enhanced, by the data. At large backward angles with $\theta_{\text {c.m. }} \gtrsim 145^{\circ}$, calculations 


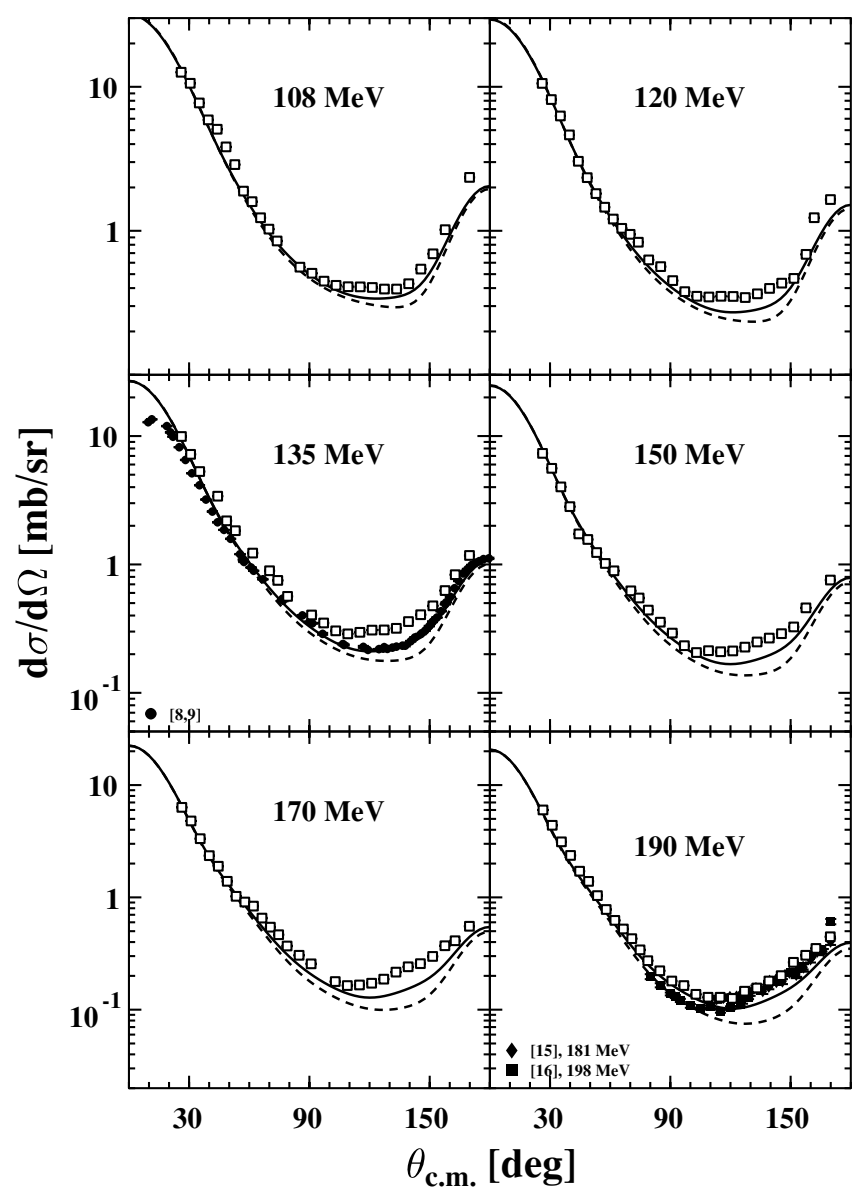

FIG. 10. Differential cross section for the reaction ${ }^{2} \mathrm{H}(\vec{p}, d p)$ as a function of incident-beam energy. The data set measured in this work is denoted by open squares. The statistical uncertainty is depicted at each data point. Also shown are the results of the calculations outlined in Sec. II B using CD-Bonn (dashed line) and $\mathrm{CD}-$ Bonn $+\Delta$ (solid line). The region of the minimum is shown in Fig. 8.

using either form of $3 N F$ describe our data reasonably well at energies up to $135 \mathrm{MeV}$. However, with increasing bombarding energy, discrepancies in the calculations set in at backward angles around $\theta_{\text {c.m. }} \approx 130^{\circ}$.

The measured vector analyzing powers of the ${ }^{2} \mathrm{H}(\vec{p}, d p)$ reaction at six incident-beam energies are shown in Figs. 12 through 16. Figure 12 presents, in addition to the present data, the results of the measurement by Wells et al. at $120 \mathrm{MeV}$ [17] and Bieber et al. at $150 \mathrm{MeV}$ and $190 \mathrm{MeV}$ [18]. At $150 \mathrm{MeV}$, data from Kuroda et al. at $155 \mathrm{MeV}$ [14] and Postma and Wilson at $146 \mathrm{MeV}$ [13] are shown as well. These two data sets have rather large statistical uncertainties. At $190 \mathrm{MeV}$, results from Adelberger and Brown [16] and Cadman et al. [19] are also shown. As for the cross-section results, the data at $217 \mathrm{MeV}$ from Ref. [15] have been omitted at $190 \mathrm{MeV}$, because the difference in kinetic energy is too large for a meaningful comparison. The same holds for the Saturne measurements from reference [63]. At all energies except for 120 and $190 \mathrm{MeV}$, a high-precision measurement

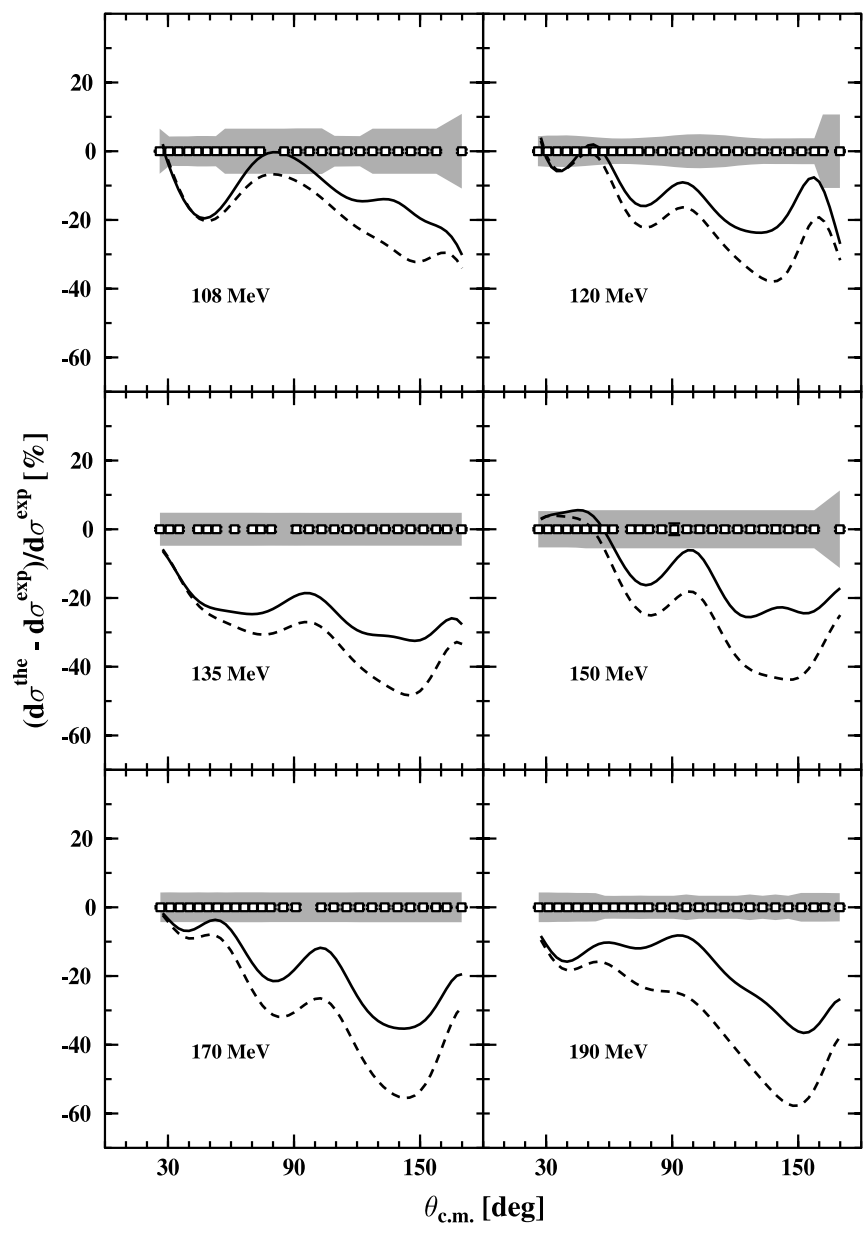

FIG. 11. Shown are the deviations of the theoretical calculations shown in Fig. 10 from the data set for differential cross sections of the reaction ${ }^{2} \mathrm{H}(\vec{p}, d p)$ measured in this work. Further information about the presentation is given in the text. The meaning of the curves is the same as described in the legend to Fig. 10. The gray band represents the systematic uncertainty of the measurement.

at $\theta_{\text {c.m. }} \approx 90^{\circ}$ from IUCF [20] is shown. As can be seen in Fig. 12, the measured data from this work agree very well with the other data sets. However, in contrast to the other data sets, the high-precision data measured in this work cover, on their own, a large range in kinetic energy as well as a broad region in c.m. angles. Again, to see the differences better at large angles, the region between $90^{\circ}$ and $150^{\circ}$ is shown on a larger scale in Fig. 13.

The black band in Figs. 12-14 at each energy represents results of calculations using $N N$ potentials only, whereas the gray band shows the results from $N N+$ TM99 calculations. The solid line again shows the results of calculations using the AV18+Urbana-IX potential. The deviations of the theoretical calculations from the present data are shown in Fig. 14. The meaning of the theoretical curves is the same as for Fig. 12. In Fig. 14 (and also 16), the data from this work are set to zero and are marked with their statistical uncertainties. The systematic uncertainty of $3 \%$ is shown as a band around these data points. 


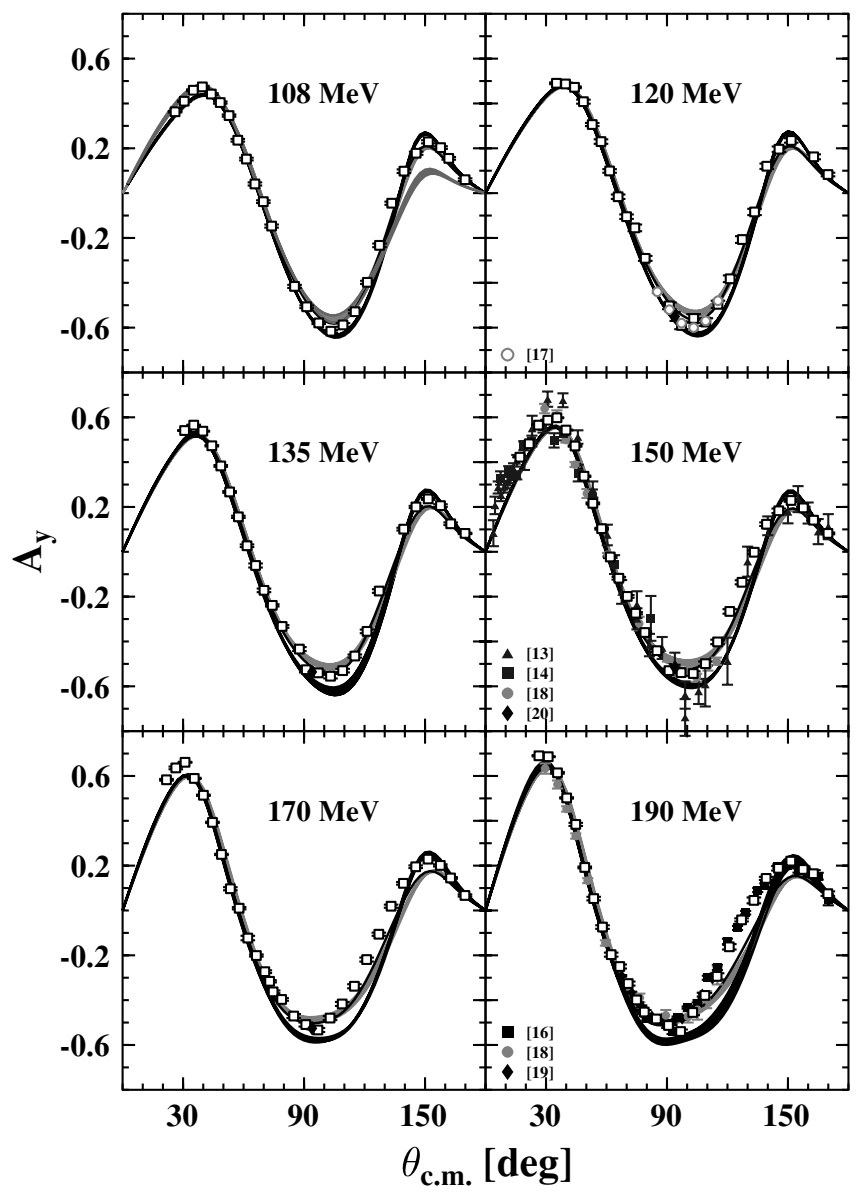

FIG. 12. Results for the vector analyzing power of the ${ }^{2} \mathrm{H}(\vec{p}, d p)$ reaction. The results of the present work are shown by open squares, whereas other symbols represent data available in the literature. The theoretical predictions are shown for $N N$ potentials only (black band), $N N+$ TM99 interactions (gray band), and a calculation from AV18+Urbana-IX (solid line), which is hard to distinguish from the gray band. At $108 \mathrm{MeV}$, the results of the $\chi \mathrm{PT}$ calculations are shown by dark gray band, which is very close to the gray band. For each data point, only the statistical uncertainty is given.

Comparing the calculations and the data separately for each energy, it can be observed that the theoretical predictions deviate slightly from the data below a center-of-mass angle of $40^{\circ}$. This might be because of Coulomb-effects, although the deviation seems to get larger in this range as one increases the beam energy. It might also be an onset of relativistic effects, which are not properly included in the theory. In the angular range between $40^{\circ} \lesssim \theta_{\text {c.m. }} \lesssim 60^{\circ}$, depending on the bombarding energy, $N N$ - and $(N N+3 N)$ calculations agree with each other and describe the data reasonably well. At $\theta_{\text {c.m. }} \approx 50^{\circ}$, calculations with and without $3 \mathrm{NF}$ start to deviate from each other. Calculations using $N N$ potentials only fail to describe the data. With the use of the modified TucsonMelbourne (TM) force or the Urbana-IX potential, these discrepancies can be remedied partly. However, at backward

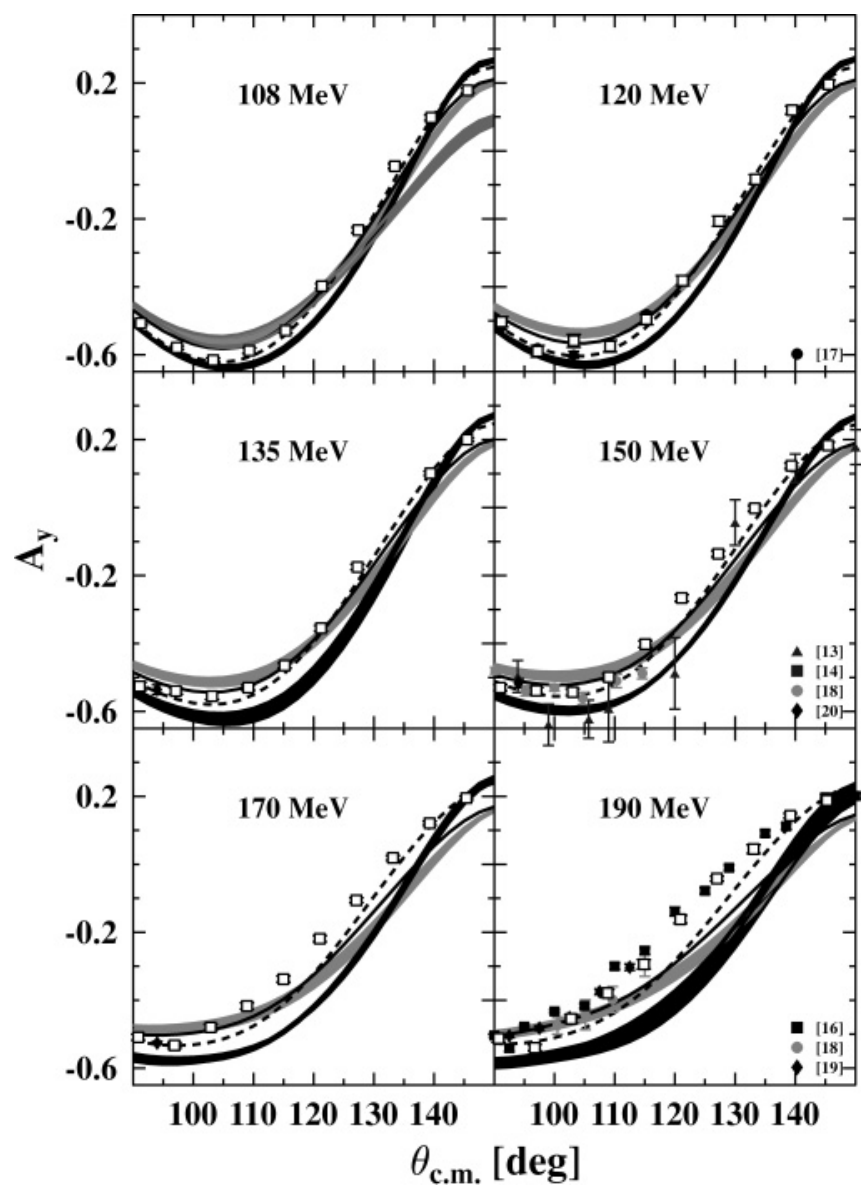

FIG. 13. The vector analyzing powers for the angular range between $90^{\circ}$ and $150^{\circ}$ on a larger scale as compared to that of Fig. 12. For explanation of curves and data, see Fig. 12 with the lower gray band in the $108-\mathrm{MeV}$ panel from the $\chi \mathrm{PT}$ calculations. The extra curve shown by the dashed line is for CD-Bonn $+\Delta$ of Fig. 15.

angles around $\theta_{\text {c.m. }} \approx 130^{\circ}$, also these models fail to describe the data, as can be seen very clearly in Fig. 14.

Looking at the behavior of $A_{y}$ as a function of energy, it can be observed, that the first maximum and the minimum shift toward smaller angles with increasing energy, whereas the second maximum stays at around $\theta_{\text {c.m. }} \approx 150^{\circ}$. The minimum of the calculations also becomes shallower with increasing incident energy, whereas the minimum of the experimental data hardly changes its form. As can be observed from Fig. 14, this behavior leads to large disagreements between the calculations and the data at higher energies and backward angles, which are of the same order as the deviations of calculations using $N N$ potentials only. Interesting to note is the fact that calculations from AV18+Urbana-IX, which are based for a large part on phenomenology, still come closer to the present data at higher energies than calculations that employ the modified Tuscon-Melbourne force, TM99, which is a theoretical model obeying chiral symmetry. 


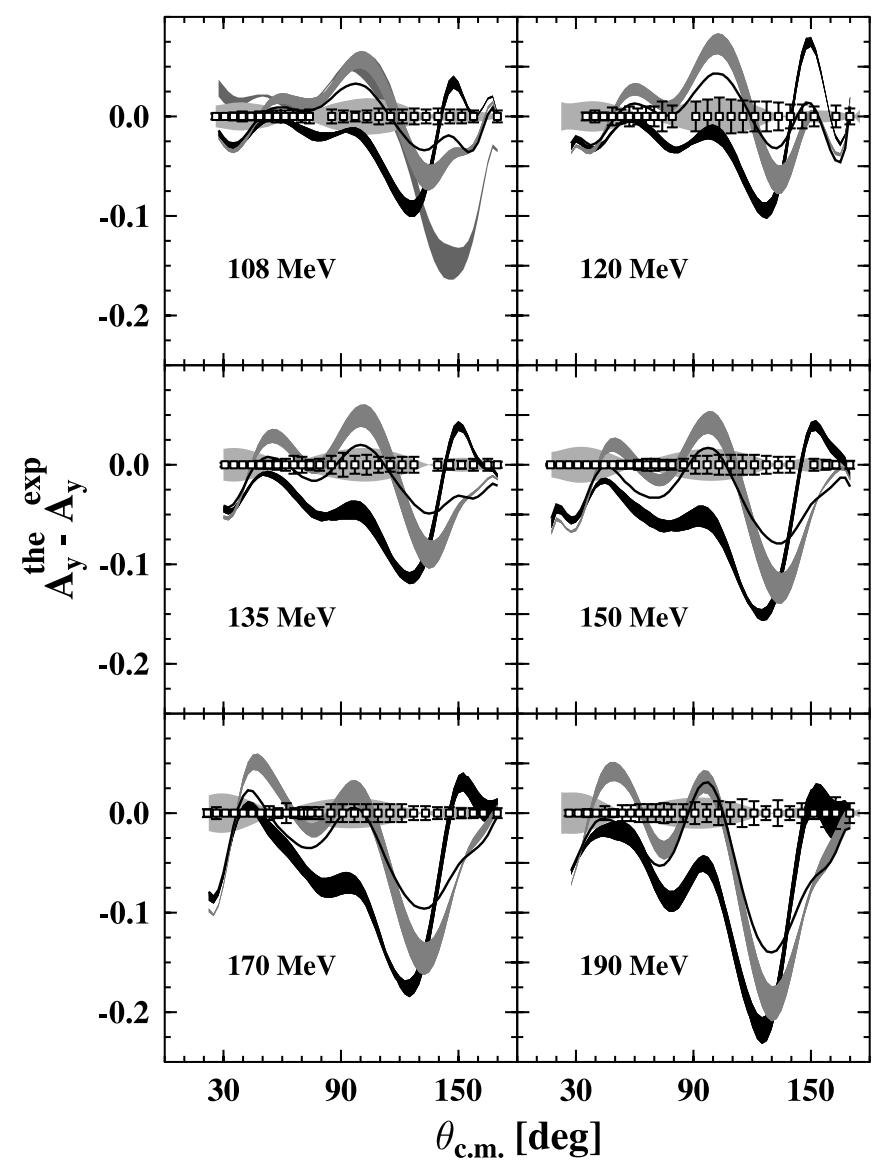

FIG. 14. Differences between various theoretical predictions and experimental analyzing powers of the reaction ${ }^{2} \mathrm{H}(\vec{p}, d p)$ measured in this work. Further information about the presentation of the data and the calculations is given in the text. The meaning of the curves is the same as described in the legend to Fig. 12.

In Figs. 15 and 16, the same observables as in Figs. 12 and 14 are shown with results from calculations done in the framework outlined in Sec. IIB. These figures are shown separately to avoid having too many curves in one picture. Also, the data sets from Refs. [13] and [14] have been omitted in these figures for clarity. Calculations from the purely nucleonic CD-Bonn potential (dashed curve) and from its coupled-channel extension CD-Bonn $+\Delta$ (solid curve) are compared. As can be observed, calculations using explicit $\Delta$ excitations as mediator for an effective $3 N F$ describe our data rather well for incident-beam energies up to $150 \mathrm{MeV}$ over a large angular range in the center-of-mass frame of reference. Only at 170 and $190 \mathrm{MeV}$ do deviations start to set in at the same place, where the deficiencies of TM99 and Urbana-IX are observed (i.e., at around $\theta_{\text {c.m. }} \approx 130^{\circ}$ ). Deviations can be seen at very forward angles, too, which, again, may be because of Coulomb effects. Comparing Figs. 14 and 16 , it can be observed that the calculations that use an explicit $\Delta$ excitation give a better description of the analyzing-power data. In fact, up to a bombarding energy of $135 \mathrm{MeV}$, the results of the calculations based on the framework outlined in Sec. II B are within the uncertainties of

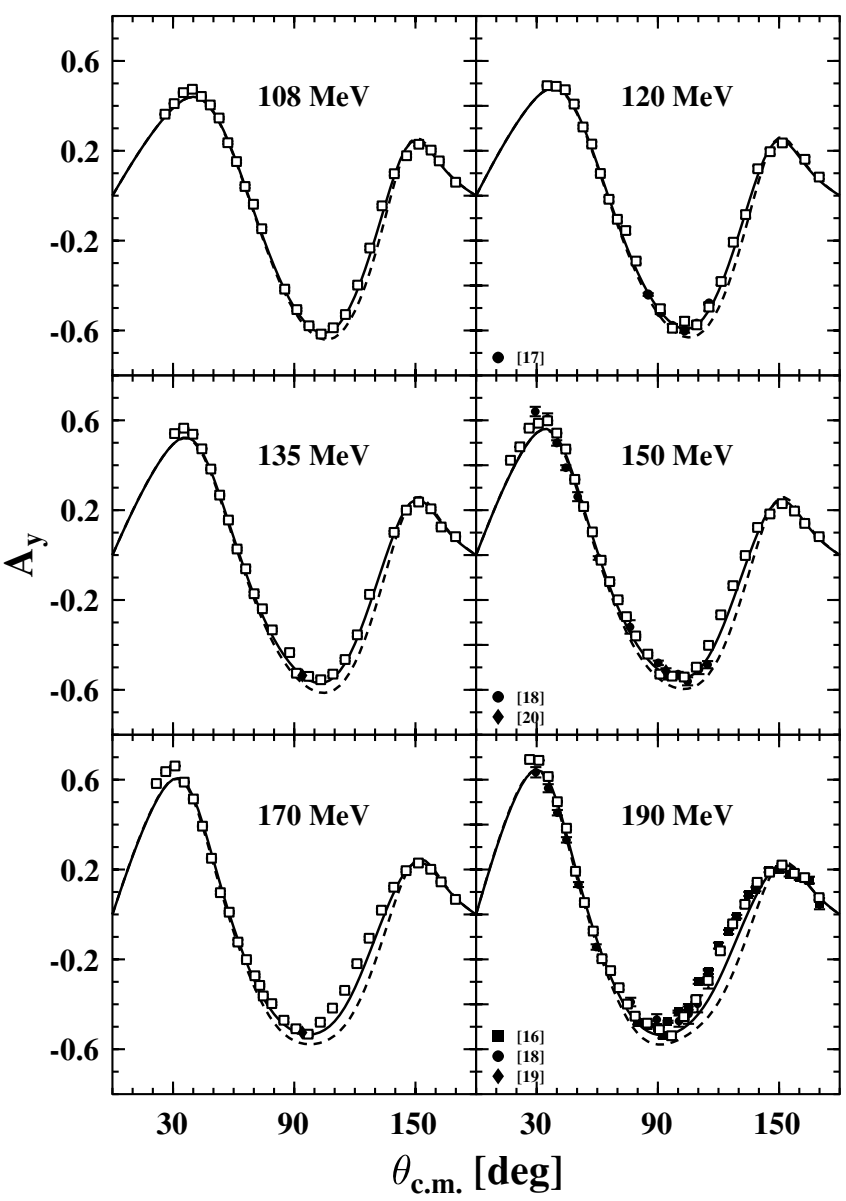

FIG. 15. Results for the vector analyzing power of the ${ }^{2} \mathrm{H}(\vec{p}, d p)$ reaction in comparison with coupled-channel calculations using the potentials CD-Bonn (dashed line) and CD-Bonn $+\Delta$ (solid line). The statistical uncertainty is given for each data point. The region of the minimum is shown in Fig. 13.

the data measured in this work. But also at the higher energies, the predictions from these calculations lie closer to our data than the predictions that use TM99 or Urbana-IX. The two sets of calculations using $N N$ forces only agree well with each other showing the numerical robustness of the computations. For $A_{y}$, the calculations using explicit $\Delta$ excitations agree better with the data than the calculations using the three-nucleon forces. However, for the cross sections, both calculations do equally poorly at backward angles. This indicates that in both approaches, additional dynamical ingredients are required. Although the coupled-channel calculations of the Sec. II B seem to be in better agreement with our data, this does not necessarily imply that they are more complete than the calculations outlined in Sec. A. Note that the results of all calculations using $N N$ potential agree very well only with each other.

The behavior of both, the analyzing power and the differential cross section, at a typical backward angle is shown in Fig. 17. Here, both observables and the deviations of the calculations from our data are plotted as a function of incident-beam energy for $\theta_{\mathrm{d}, \text { lab }}=20^{\circ}$, which corresponds to 


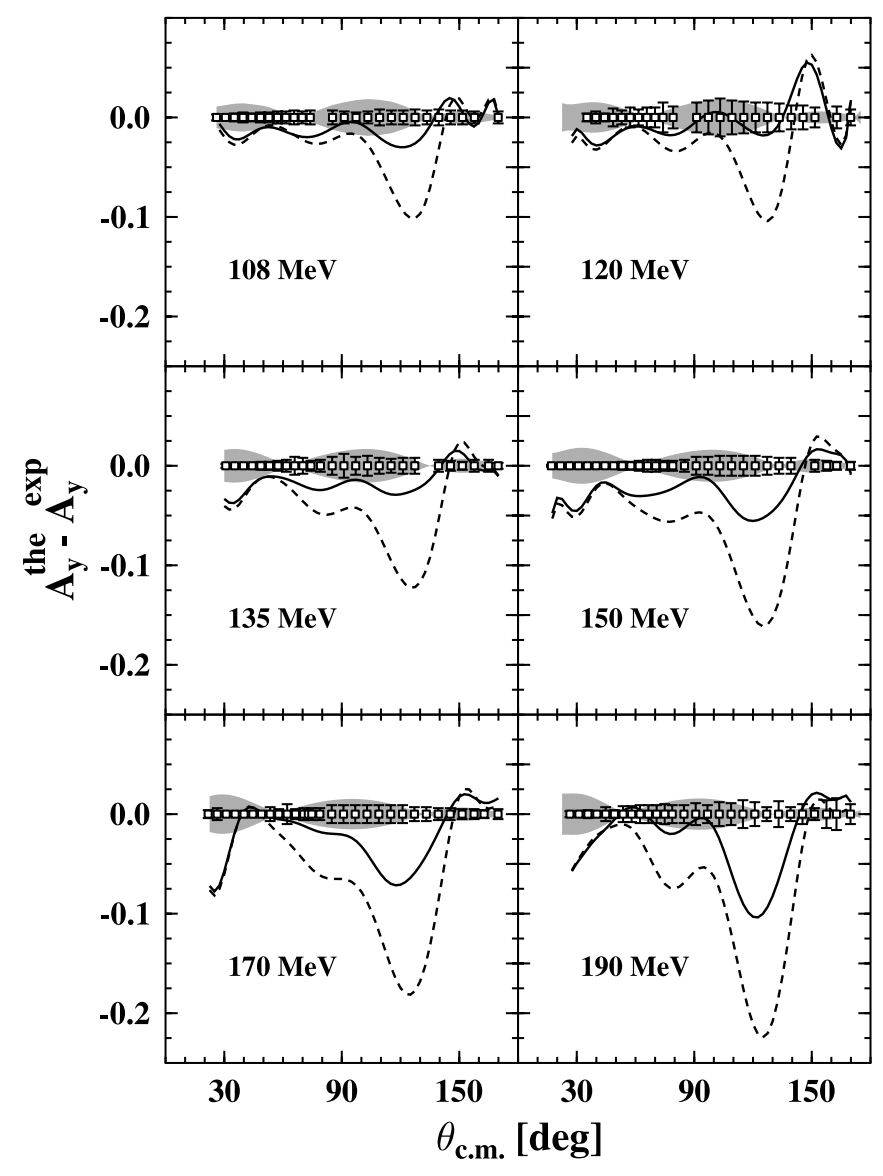

FIG. 16. Shown are the deviations of the theoretical calculations from the measured analyzing powers of the reaction ${ }^{2} \mathrm{H}(\vec{p}, d p)$ shown in Fig. 15. The meaning of the curves is the same as described in the legend to Fig. 15. Further information about the presentation of the data and the calculations is given in the text.

$\theta_{\text {c.m. }} \approx 139^{\circ}$. Further shown are the results from $N N+$ TM99, AV18+Urbana-IX, and CD-Bonn $+\Delta$. As can be observed in Fig. 17, the deviations for both observables increase at $\theta_{\text {c.m. }} \approx 139^{\circ}$ with increasing bombarding energy. Furthermore, as is stressed in Fig. 17, the spread between the different modern $3 N F$ s is, in the case of the differential cross section, negligible. However, in the case of the analyzing powers, the spread leads to a still reasonable description of the data with calculations from CD-Bonn $+\Delta$ but to rather large disagreements between the data and calculations from $N N+$ TM99 or AV18+Urbana-IX.

The differences seen between the different $3 N F$ models, shown in Figs. 9-16 and summarized, for one particular angle, in Fig. 17, seem to indicate that the different models must differ mainly in the treatment of the spin. This, however, has yet to be established because these models differ from each other in other respects as well and have in common only the approach of using a modern, phenomenological, $N N$ potential and adding a $3 N F$ to it.

The $3 N F$ TM99 is certainly the most sophisticated version of the Tuscon-Melbourne forces as it also respects chiral symmetry. The modifications with respect to the original TM force seem to influence mainly the spin-dependent part of the potential, as no significant change in the prediction for $d \sigma / d \Omega$ was observed.

In contrast, the Urbana-IX model, which was built for a completely different purpose, is based largely on phenomenology. However, whereas calculations from the two forces agree for the differential cross section, calculations from AV18+Urbana-IX give a slightly better description of the analyzing power. Calculations based on an explicit $\Delta$ excitation seem to give the best description of the analyzing power; it is unclear, if that good description is accidental or if the retarded $\Delta$-isobar propagation and/or the included heavier meson exchanges are the dynamic reason.

The common discrepancies with our data at backward angles around $\approx 130^{\circ}-150^{\circ}$ at higher incident-beam energies, which is a kinematical region with large momentum transfer, seem to indicate common deficiencies in the proper inclusion of relativistic dynamics. It might also be a hint of deficiencies in the treatment of the spin-dependent part at higher momentum transfer, and/or because of higher order effects that have not been included in the calculations, such as $\rho-\pi$ or $\rho-\rho$ exchanges in the case of TM99 and Urbana-IX forces.

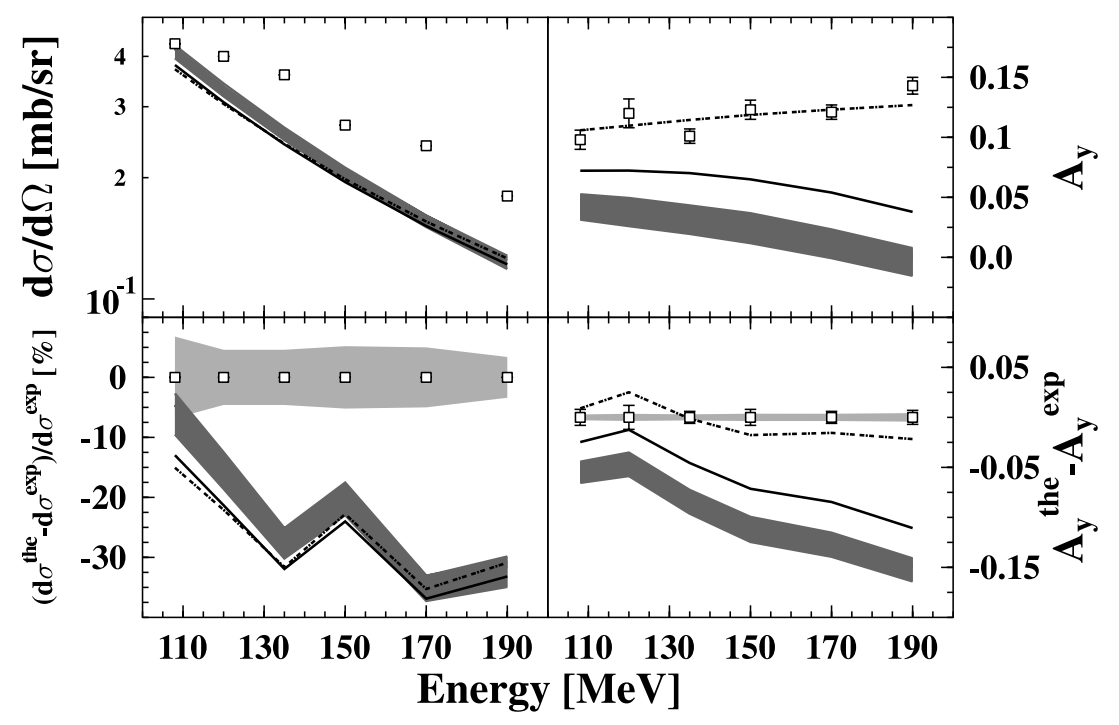

FIG. 17. Comparison of theoretical calculations for $d \sigma / d \Omega$ and $A_{y}$ for the reaction ${ }^{2} \mathrm{H}(\vec{p}, d p)$ with measured values as a function of energy for $\theta_{\text {c.m. }} \approx 139^{\circ}\left(\theta_{\mathrm{d} \text { lab }}=20^{\circ}\right)$. Shown are calculations from $N N+$ TM99 (gray band), AV18+Urbana-IX (solid line), and CD-Bonn $+\Delta$ $(\cdot-\cdot$ line). The systematic uncertainty of the data set is shown in the lower panels as a gray band around zero. 
The results from $\chi \mathrm{PT}$ at $108 \mathrm{MeV}$ incident-beam energy give a reasonable description for the differential cross section, but show deficiencies in the description of the analyzing power at backward angles. However, these calculations were only done up to NNLO in $\chi \mathrm{PT}$. In this order, $3 N F$ appear for the first time. To guarantee convergence, higher order terms should be included that might also remove the discrepancies with the data.

\section{SUMMARY AND CONCLUSIONS}

In this article, one of the most complete measurements for the cross sections and analyzing powers of the elastic scattering of protons from deuterons at intermediate energies has been presented. The experiments were performed at KVI using the superconducting cyclotron AGOR, with polarized proton beams with energies ranging from 108 to $190 \mathrm{MeV}$. The polarization of the beam was measured and monitored continuously during the data taking with the IBP with a systematic uncertainty of around $3 \%$. The statistical uncertainty of the measurements were generally much smaller than this value.

The BBS with its focal-plane detection system was used for the cross-section and analyzing-power measurements. This magnetic device was placed at laboratory angles between $5^{\circ}$ and $50^{\circ}$ for the detection of protons and deuterons such that a center-of-mass angle coverage from $30^{\circ}$ to $170^{\circ}$ was guaranteed. Solid $\mathrm{C}_{2} \mathrm{D}_{4} / \mathrm{C}_{2} \mathrm{H}_{4}$ targets were used, where the $\mathrm{C}_{2} \mathrm{H}_{4}$ component was exploited to extract the target thickness information from proton-proton elastic scattering. A coincidence detector inside the scattering chamber was used to detect the recoil particles for some angles where more was needed to identify the event. Most of the runs were, however, taken with BBS alone where there was a background from the carbon target in the spectrometer. This background was subtracted through a fitting procedure. Runs with pure carbon and comparisons to runs where the coincidence detector was used showed that the procedure was very effective.

The number of counts in the detector were converted to cross sections and analyzing powers having corrected for inefficiencies of the detector and computer dead time. These results are presented in Figs. 7 through 16 and the tables in the appendix. As can be observed in the figures, it is only with the present statistical and systematic uncertainties that one can make a sensible study of possible three-nucleon force $(3 N F)$ effects. From Figs. 9, 11, 14, and 16, one can see a systematic increase in the deviation between the theories and the experimental data as a function of energy, with a possible exception of the results of the coupled-channel calculations outlined in Sec. II B for the analyzing powers. These deviations that are largest for the backward angles are summarized in Fig. 17 for a typical backward angle. This trend points to the fact that the $3 N F$ s used in the calculations are not yet well understood. It is also possible that relativity becomes more important at these large momentum transfers. This effect is being studied presently [64]. It is also possible that heavier mesons, which should gain importance at higher momenta, must be included in the treatment of the $3 N F$ of the $3 N$ Hilbert-space approach. This has been explicitly included in the calculations outlined in Sec. II B. This might be one reason why this calculation performs better for the analyzing powers even though it is not clear why it is doing the same as the others for the cross sections.

The calculation within the $\chi$ PT have been performed only for the lowest energy and to the NNLO. These calculations should be pursued further as the two- and the $3 \mathrm{NF}$ are treated on the same footing. However, to guarantee convergence, higher orders should be included in the calculations before definitive conclusions can be made.

In general, it can be concluded that the treatment of threenucleon observables, such as $d \sigma / d \Omega$ or $A_{y}$, using the approach of adding a $3 N F$ to a phenomenological $N N$ potential seems to work well at lower kinetic energies. However, for higher kinetic energies, and for backward angles, more sophisticated approaches with other types of $3 \mathrm{NF}$ s are necessary. This calls for further theoretical investigations.

\section{ACKNOWLEDGMENTS}

The authors thank H. Meijer and his group at the University of Groningen for the careful determination of the relative abundance of hydrogen and deuterium in the targets. This work was performed as part of the research program of the Stichting voor Fundamenteel Onderzoek der Materie (FOM) with financial support from the Nederlandse Organisatie voor Wetenschappelijk Onderzoek (NWO) and was supported by the Deutsche Forschungsgemeinschaft, the Polish Committee for Scientific Research under Grant No. 2P03B00825, the NATO Grant No. PST.CLG.978943, and DOE Grant Nos. DE-FC02-01ER41187 and DE-FG03-00ER41132. The numerical calculations of the Bochum-Cracow group have been performed on the Cray T90 and T3E of the NIC in Jülich, Germany. 


\section{APPENDIX: DATA TABLES}

TABLE I. Results for the vector analyzing power $A_{y}$ and the differential cross section $d \sigma / d \Omega$ at $108 \mathrm{MeV}$ incident-beam energy. The angles are given in [deg]. $d \sigma$ is an abbreviation for $d \sigma / d \Omega$ given in units of $\mathrm{mb} / \mathrm{sr}$. The subscripts $p, d$ at $\theta_{\text {lab }}$ denote the particle (proton or deuteron) that was measured with the BBS/ESN. The statistical uncertainties are given by $\Delta d \sigma_{\mathrm{S}}=\Delta(d \sigma / d \Omega)_{\text {stat }}$ and $\Delta A_{y, \mathrm{~S}}=$ $\Delta A_{y \text {,stat }}$ (as absolute value). All systematic uncertainties, $\Delta d \sigma_{\mathrm{P}}=$ $\Delta(d \sigma / d \Omega)_{\mathrm{pol}}, \Delta d \sigma_{N}=\Delta(d \sigma / d \Omega)_{\mathrm{norm}}$, and $\Delta d \sigma_{T}$, are given in percentages. $\Delta d \sigma_{N}$ includes the uncertainties from proton-proton scattering and from the VDC-efficiency correction. The column $\Delta d \sigma_{T}$ contains the squared sums of $\Delta d \sigma_{P}$ and $\Delta d \sigma_{N}$. An additional systematic point-to-point uncertainty of $4 \%$ should be added to the statistical uncertainty, as discussed in Sec. IV D. The total systematic uncertainty for the cross section is then $<7 \%$. For the analyzing powers, a systematic uncertainty of around $3 \%$ should be taken for all the points.

\begin{tabular}{rrrrrrrrr}
\hline \hline$\theta_{\text {lab }}$ & $\theta_{\text {c.m. }}$ & $A_{y}$ & $\Delta A_{y, \mathrm{~S}}$ & $d \sigma$ & $\Delta d \sigma_{\mathrm{S}}$ & $\Delta d \sigma_{P}$ & $\Delta d \sigma_{N}$ & $\Delta d \sigma_{T}$ \\
\hline $17_{p}$ & 26.03 & 0.363 & 0.003 & 12.619 & 0.021 & 0.6 & 5.2 & 5.2 \\
$20_{p}$ & 30.57 & 0.410 & 0.003 & 10.575 & 0.023 & 0.8 & 1.2 & 1.4 \\
$23_{p}$ & 35.09 & 0.459 & 0.004 & 7.725 & 0.026 & 0.9 & 1.2 & 1.5 \\
$26_{p}$ & 39.57 & 0.474 & 0.005 & 5.894 & 0.024 & 1.0 & 1.2 & 1.6 \\
$29_{p}$ & 44.02 & 0.442 & 0.004 & 5.073 & 0.018 & 0.8 & 1.7 & 1.9 \\
$32_{p}$ & 48.43 & 0.404 & 0.005 & 3.826 & 0.016 & 0.8 & 1.7 & 1.9 \\
$35_{p}$ & 52.80 & 0.346 & 0.005 & 2.883 & 0.013 & 0.7 & 1.7 & 1.8 \\
$38_{p}$ & 57.13 & 0.236 & 0.006 & 1.886 & 0.009 & 0.4 & 5.2 & 5.2 \\
$41_{p}$ & 61.41 & 0.152 & 0.006 & 1.594 & 0.005 & 0.3 & 5.2 & 5.2 \\
$44_{p}$ & 65.63 & 0.041 & 0.007 & 1.232 & 0.005 & 0.1 & 5.2 & 5.2 \\
$47_{p}$ & 69.80 & -0.038 & 0.006 & 1.027 & 0.004 & 0.1 & 5.2 & 5.2 \\
$50_{p}$ & 73.90 & -0.147 & 0.007 & 0.850 & 0.003 & 0.3 & 5.2 & 5.2 \\
$47_{d}$ & 85.27 & -0.416 & 0.007 & 0.559 & 0.002 & 0.8 & 5.2 & 5.3 \\
$44_{d}$ & 91.27 & -0.507 & 0.006 & 0.507 & 0.002 & 1.0 & 5.2 & 5.3 \\
$41_{d}$ & 97.28 & -0.579 & 0.005 & 0.447 & 0.002 & 1.1 & 5.2 & 5.3 \\
$38_{d}$ & 103.29 & -0.616 & 0.006 & 0.418 & 0.002 & 1.2 & 5.2 & 5.3 \\
$35_{d}$ & 109.31 & -0.588 & 0.008 & 0.409 & 0.003 & 1.1 & 1.6 & 1.9 \\
$32_{d}$ & 115.34 & -0.529 & 0.007 & 0.408 & 0.003 & 1.0 & 1.6 & 1.9 \\
$29_{d}$ & 121.38 & -0.398 & 0.007 & 0.404 & 0.002 & 0.8 & 1.6 & 1.8 \\
$26_{d}$ & 127.42 & -0.233 & 0.008 & 0.394 & 0.002 & 0.5 & 5.2 & 5.2 \\
$23_{d}$ & 133.47 & -0.045 & 0.007 & 0.394 & 0.002 & 0.1 & 5.2 & 5.2 \\
$20_{d}$ & 139.53 & 0.098 & 0.008 & 0.429 & 0.002 & 0.2 & 5.2 & 5.2 \\
$17_{d}$ & 145.59 & 0.178 & 0.007 & 0.542 & 0.003 & 0.3 & 5.2 & 5.2 \\
$14_{d}$ & 151.66 & 0.229 & 0.007 & 0.692 & 0.004 & 0.4 & 5.2 & 5.2 \\
$11_{d}$ & 157.73 & 0.203 & 0.006 & 1.018 & 0.004 & 0.3 & 5.2 & 5.2 \\
$5_{d}$ & 169.87 & 0.060 & 0.006 & 2.349 & 0.012 & 0.1 & 10.1 & 10.1 \\
\hline \hline & & & & & & & & \\
\hline \hline
\end{tabular}

TABLE II. Results for the vector analyzing power $A_{y}$ and the differential cross section at $120 \mathrm{MeV}$ incident-beam energy. The symbols and the units are the same as in Table I. The analyzing powers given in this table are from [21] and were measured independently from the differential cross section from Ref. [22]. The systematic point-to-point uncertainty, as discussed in Sec. IV D, at this energy is $3.5 \%$. The total systematic uncertainty is, therefore, $<5 \%$. For the analyzing powers, a systematic uncertainty of around $3 \%$ should be taken for all the points. A few numbers shown in italics for the analyzing powers were obtained in a separate measurement than the rest of the analyzing powers, whereby the systematic errors were around $8 \%$. These points are not plotted in the figures.

\begin{tabular}{rrrlllllll}
\hline \hline$\theta_{\text {lab }}$ & $\theta_{\text {c.m. }}$ & $A_{y}$ & $\Delta A_{y, S}$ & \multicolumn{1}{c}{$d \sigma$} & $\Delta d \sigma_{S}$ & $\Delta d \sigma_{P}$ & $\Delta d \sigma_{N} \Delta d \sigma_{T}$ \\
\hline $17_{p}$ & 26.10 & 0.434 & 0.004 & 10.558 & 0.026 & 2.3 & 1.2 & 2.6 \\
$20_{p}$ & 30.65 & 0.467 & 0.003 & 8.156 & 0.018 & 2.6 & 1.3 & 2.9 \\
$23_{p}$ & 35.17 & 0.490 & 0.004 & 6.274 & 0.02 & 2.6 & 1.3 & 2.9 \\
$26_{p}$ & 39.67 & 0.487 & 0.006 & 4.635 & 0.009 & 2.7 & 1.3 & 3.0 \\
$29_{p}$ & 44.13 & 0.472 & 0.004 & 3.039 & 0.007 & 2.5 & 1.2 & 2.8 \\
$32_{p}$ & 48.55 & 0.408 & 0.009 & 2.34 & 0.007 & 2.2 & 1.2 & 2.5 \\
$35_{p}$ & 52.93 & 0.306 & 0.007 & 1.813 & 0.006 & 1.7 & 1.2 & 2.1 \\
$38_{p}$ & 57.26 & 0.230 & 0.01 & 1.458 & 0.005 & 1.2 & 1.2 & 1.7 \\
$41_{p}$ & 61.54 & 0.099 & 0.008 & 1.207 & 0.004 & 0.5 & 1.2 & 1.3 \\
$44_{p}$ & 65.77 & -0.016 & 0.01 & 1.04 & 0.004 & 0.2 & 1.2 & 1.2 \\
$47_{p}$ & 69.94 & -0.105 & 0.01 & 0.945 & 0.004 & 0.6 & 1.2 & 1.3 \\
$50_{p}$ & 74.05 & -0.155 & 0.015 & 0.833 & 0.003 & 1.0 & 1.2 & 1.6 \\
$50_{d}$ & 79.20 & -0.291 & 0.011 & 0.628 & 0.002 & 1.7 & 1.1 & 2.0 \\
$47_{d}$ & 85.19 & -0.390 & 0.006 & 0.564 & 0.002 & 2.1 & 1.1 & 2.4 \\
$44_{d}$ & 91.19 & -0.503 & 0.015 & 0.451 & 0.003 & 2.7 & 1.1 & 2.9 \\
$41_{d}$ & 97.20 & -0.590 & 0.017 & 0.378 & 0.002 & 3.2 & 1.1 & 3.4 \\
$38_{d}$ & 103.21 & -0.559 & 0.019 & 0.351 & 0.001 & 3.3 & 1.1 & 3.5 \\
$35_{d}$ & 109.24 & -0.575 & 0.017 & 0.347 & 0.002 & 3.1 & 1.2 & 3.3 \\
$32_{d}$ & 115.27 & -0.496 & 0.016 & 0.351 & 0.001 & 2.7 & 1.2 & 3.0 \\
$29_{d}$ & 121.31 & -0.382 & 0.015 & 0.35 & 0.001 & 2.0 & 1.2 & 2.3 \\
$26_{d}$ & 127.36 & -0.207 & 0.015 & 0.344 & 0.002 & 1.2 & 1.2 & 1.7 \\
$23_{d}$ & 133.42 & -0.084 & 0.014 & 0.365 & 0.002 & 0.3 & 1.2 & 1.2 \\
$20_{d}$ & 139.48 & 0.120 & 0.012 & 0.398 & 0.002 & 0.5 & 1.2 & 1.3 \\
$17_{d}$ & 145.55 & 0.196 & 0.012 & 0.433 & 0.002 & 0.6 & 1.2 & 1.3 \\
$14_{d}$ & 151.62 & 0.235 & 0.01 & 0.467 & 0.002 & 0.8 & 1.2 & 1.4 \\
$11_{d}$ & 157.70 & 0.217 & 0.007 & 0.686 & 0.004 & 0.7 & 1.2 & 1.4 \\
$8.5_{d}$ & 162.76 & 0.162 & 0.011 & 1.233 & 0.002 & 0.5 & 10.1 & 10.1 \\
$5_{d}$ & 169.86 & 0.083 & 0.008 & 1.648 & 0.005 & 0.2 & 10.1 & 10.1 \\
\hline \hline & & & & & & & & \\
\hline
\end{tabular}


TABLE III. Results for the vector analyzing power $A_{y}$ and the differential cross section $d \sigma / d \Omega$ at $135 \mathrm{MeV}$ incident-beam energy. The notation for the symbols and the units are the same as in Table I. The differential cross section was measured with an unpolarized beam using the CUSP source, whereas $A_{y}$ was measured at a different time using POLIS. The systematic point-to-point uncertainty, as discussed in Sec. IVD, is $4.5 \%$ at this energy, resulting in a total systematic uncertainty of $<5 \%$. For the analyzing powers, a systematic uncertainty of around $3 \%$ should be taken for all the points.

\begin{tabular}{rrrcccc}
\hline \hline$\theta_{\text {lab }}$ & $\theta_{\text {c.m. }}$ & $A_{y}$ & $\Delta A_{y, S}$ & $d \sigma$ & $\Delta d \sigma_{S}$ & $\Delta d \sigma_{N}$ \\
\hline $17_{p}$ & 26.18 & - & - & 9.922 & 0.039 & 1.7 \\
$20_{p}$ & 30.75 & 0.541 & 0.002 & 7.204 & 0.020 & 1.7 \\
$23_{p}$ & 35.28 & 0.565 & 0.002 & 5.292 & 0.027 & 1.7 \\
$26_{p}$ & 39.79 & 0.538 & 0.002 & - & - & - \\
$29_{p}$ & 44.26 & 0.473 & 0.003 & 3.401 & 0.013 & 1.7 \\
$32_{p}$ & 48.69 & 0.383 & 0.004 & 2.186 & 0.016 & 1.7 \\
$35_{p}$ & 53.08 & 0.267 & 0.004 & 1.832 & 0.011 & 1.7 \\
$38_{p}$ & 57.42 & 0.156 & 0.005 & - & - & - \\
$41_{p}$ & 61.71 & 0.027 & 0.006 & 1.226 & 0.008 & 1.7 \\
$44_{p}$ & 65.95 & -0.061 & 0.009 & - & - & - \\
$47_{p}$ & 70.12 & -0.172 & 0.008 & 0.893 & 0.004 & 1.7 \\
$50_{p}$ & 74.23 & -0.239 & 0.005 & 0.751 & 0.005 & 1.6 \\
$50_{d}$ & 79.11 & -0.333 & 0.006 & 0.564 & 0.004 & 1.6 \\
$47_{d}$ & 87.69 & -0.434 & 0.009 & - & - & - \\
$44_{d}$ & 91.09 & -0.526 & 0.012 & 0.404 & 0.004 & 1.6 \\
$41_{d}$ & 97.10 & -0.540 & 0.009 & 0.350 & 0.002 & 1.6 \\
$38_{d}$ & 103.12 & -0.555 & 0.010 & 0.304 & 0.002 & 1.6 \\
$35_{d}$ & 109.14 & -0.530 & 0.008 & 0.288 & 0.001 & 1.6 \\
$32_{d}$ & 115.18 & -0.465 & 0.007 & 0.296 & 0.002 & 1.6 \\
$29_{d}$ & 121.23 & -0.355 & 0.008 & 0.308 & 0.001 & 1.6 \\
$26_{d}$ & 127.28 & -0.175 & 0.008 & 0.309 & 0.001 & 1.6 \\
$23_{d}$ & 133.34 & - & - & 0.318 & 0.001 & 1.6 \\
$20_{d}$ & 139.41 & 0.101 & 0.006 & 0.359 & 0.002 & 1.6 \\
$17_{d}$ & 145.49 & 0.200 & 0.005 & 0.406 & 0.001 & 1.6 \\
$14_{d}$ & 151.57 & 0.236 & 0.004 & 0.476 & 0.002 & 1.6 \\
$11_{d}$ & 157.66 & 0.206 & 0.006 & 0.626 & 0.001 & 1.6 \\
$9_{d}$ & 162.73 & 0.125 & 0.006 & 0.832 & 0.002 & 1.6 \\
$5_{d}$ & 169.84 & 0.082 & 0.003 & 1.175 & 0.004 & 1.6 \\
\hline \hline & & & & & & \\
\hline
\end{tabular}

TABLE IV. Results for the vector analyzing power $A_{y}$ and the differential cross section at $150 \mathrm{MeV}$ incident-beam energy. The symbols and the units are the same as in Table I. The analyzing powers given in this table are from Ref. [21] and were measured independently from the differential cross section from Ref. [22]. The additional point-to-point systematic uncertainty, as discussed in Sec. IV D, is $5 \%$ at this energy, from which a total systematic uncertainty of $<6 \%$ is obtained. For the analyzing powers, a systematic uncertainty of around $3 \%$ should be taken for all the points.

\begin{tabular}{rrrrrrrrrr}
\hline \hline$\theta_{\text {lab }}$ & $\theta_{\text {c.m. }}$ & $A_{y}$ & $\Delta A_{y, S}$ & $d \sigma$ & $\Delta d \sigma_{S}$ & $\Delta d \sigma_{P}$ & $\Delta d \sigma_{N}$ & $\Delta d \sigma_{T}$ \\
\hline $11_{p}$ & 17.04 & 0.422 & 0.001 & - & - & - & - & - \\
$14_{p}$ & 21.67 & 0.482 & 0.001 & - & - & - & - & - \\
$17_{p}$ & 26.27 & 0.565 & 0.003 & 7.330 & 0.025 & 0.6 & 1.6 & 1.7 \\
$20_{p}$ & 30.85 & 0.587 & 0.002 & 5.605 & 0.021 & 0.6 & 1.6 & 1.7 \\
$23_{p}$ & 35.40 & 0.598 & 0.002 & 4.018 & 0.016 & 0.6 & 1.6 & 1.7 \\
$26_{p}$ & 39.91 & 0.543 & 0.002 & 2.820 & 0.014 & 0.5 & 1.6 & 1.7 \\
$29_{p}$ & 44.39 & 0.472 & 0.003 & 1.730 & 0.007 & 0.4 & 1.9 & 1.9 \\
$32_{p}$ & 48.84 & 0.337 & 0.003 & 1.565 & 0.008 & 0.3 & 2.4 & 2.4 \\
$35_{p}$ & 53.23 & 0.216 & 0.004 & 1.238 & 0.007 & 0.2 & 2.4 & 2.4 \\
$38_{p}$ & 57.58 & 0.103 & 0.004 & 1.018 & 0.007 & 0.1 & 2.4 & 2.4 \\
$41_{p}$ & 61.88 & -0.023 & 0.005 & 0.892 & 0.005 & 0.1 & 2.4 & 2.4 \\
$44_{p}$ & 66.12 & -0.118 & 0.006 & - & - & - & - & - \\
$47_{p}$ & 70.30 & -0.199 & 0.006 & 0.623 & 0.004 & 0.2 & 2.4 & 2.4 \\
$50_{p}$ & 74.42 & -0.274 & 0.005 & 0.548 & 0.004 & 0.3 & 2.4 & 2.4 \\
$50_{d}$ & 79.01 & -0.360 & 0.005 & 0.441 & 0.003 & 0.4 & 2.3 & 2.3 \\
$47_{d}$ & 84.99 & -0.441 & 0.006 & 0.355 & 0.003 & 0.4 & 2.3 & 2.3 \\
$44_{d}$ & 90.99 & -0.530 & 0.009 & 0.292 & 0.005 & 0.5 & 2.3 & 2.4 \\
$41_{d}$ & 97.00 & -0.539 & 0.009 & 0.233 & 0.002 & 0.5 & 2.3 & 2.4 \\
$38_{d}$ & 103.02 & -0.543 & 0.010 & 0.206 & 0.002 & 0.5 & 2.3 & 2.4 \\
$35_{d}$ & 109.05 & -0.499 & 0.010 & 0.213 & 0.001 & 0.5 & 2.3 & 2.4 \\
$32_{d}$ & 115.09 & -0.402 & 0.010 & 0.209 & 0.002 & 0.4 & 2.4 & 2.4 \\
$29_{d}$ & 121.14 & -0.266 & 0.010 & 0.212 & 0.001 & 0.3 & 2.4 & 2.4 \\
$26_{d}$ & 127.20 & -0.136 & 0.009 & 0.227 & 0.002 & 0.2 & 2.4 & 2.4 \\
$23_{d}$ & 133.27 & -0.002 & 0.008 & 0.249 & 0.002 & 0.0 & 2.4 & 2.4 \\
$20_{d}$ & 139.35 & 0.123 & 0.008 & 0.267 & 0.003 & 0.1 & 2.4 & 2.4 \\
$17_{d}$ & 145.43 & 0.183 & 0.01 & 0.289 & 0.002 & 0.2 & 2.4 & 2.4 \\
$14_{d}$ & 151.52 & 0.229 & 0.006 & 0.326 & 0.002 & 0.2 & 2.4 & 2.4 \\
$11_{d}$ & 157.62 & 0.196 & 0.005 & 0.459 & 0.002 & 0.2 & 2.4 & 2.4 \\
$8.5_{d}$ & 162.70 & 0.141 & 0.004 & - & - & - & - & - \\
$5_{d}$ & 169.82 & 0.082 & 0.004 & 0.756 & 0.004 & 0.1 & 10.1 & 10.1 \\
\hline \hline & & & & & & & & \\
\hline \hline
\end{tabular}


TABLE V. Results for the vector analyzing power $A_{y}$ and the differential cross section $d \sigma / d \Omega$ at $170 \mathrm{MeV}$ incident-beam energy. The notation for the symbols in the header and the units are the same as in Table I. The differential cross section was measured using the CUSP source, whereas the analyzing power was measured separately using POLIS. At this incident-beam energy, the point-to-point uncertainty of the data, as discussed in Sec. IV D, results in a systematic uncertainty of $4 \%$. Therefore, the total systematic uncertainty is $<5 \%$. For the analyzing powers, a systematic uncertainty of around $3 \%$ should be taken for all the points.

\begin{tabular}{crrrccc}
\hline \hline$\theta_{\text {lab }}$ & $\theta_{\text {c.m. }}$ & $A_{y}$ & $\Delta A_{y, S}$ & $d \sigma$ & $\Delta d \sigma_{S}$ & $\Delta d \sigma_{N}$ \\
\hline $14_{p}$ & 21.76 & 0.583 & 0.004 & - & - & - \\
$17_{p}$ & 26.38 & 0.636 & 0.006 & 6.331 & 0.021 & 1.6 \\
$20_{p}$ & 30.97 & 0.661 & 0.002 & 4.791 & 0.007 & 1.7 \\
$23_{p}$ & 35.54 & 0.589 & 0.003 & 3.332 & 0.009 & 1.6 \\
$26_{p}$ & 40.08 & 0.514 & 0.003 & 2.353 & 0.008 & 1.6 \\
$29_{p}$ & 44.57 & 0.393 & 0.003 & 1.893 & 0.006 & 1.7 \\
$32_{p}$ & 49.03 & 0.250 & 0.003 & 1.390 & 0.011 & 1.6 \\
$35_{p}$ & 53.44 & 0.097 & 0.007 & 1.022 & 0.005 & 1.7 \\
$38_{p}$ & 57.80 & 0.010 & 0.005 & 0.909 & 0.007 & 1.6 \\
$41_{p}$ & 62.10 & -0.123 & 0.010 & 0.836 & 0.005 & 1.7 \\
$44_{p}$ & 66.35 & -0.201 & 0.005 & 0.654 & 0.005 & 1.7 \\
$47_{p}$ & 70.54 & -0.273 & 0.006 & 0.543 & 0.002 & 1.7 \\
$48^{2} 5_{p}$ & 72.91 & -0.316 & 0.005 & - & - & - \\
$50_{p}$ & 74.66 & -0.363 & 0.006 & 0.465 & 0.003 & 1.7 \\
$50_{d}$ & 78.88 & -0.397 & 0.006 & 0.369 & 0.002 & 1.6 \\
$47_{d}$ & 84.86 & -0.471 & 0.009 & 0.305 & 0.002 & 1.6 \\
$44_{d}$ & 90.86 & -0.509 & 0.009 & 0.256 & 0.002 & 1.6 \\
$41_{d}$ & 96.87 & -0.533 & 0.009 & - & - & - \\
$38_{d}$ & 102.89 & -0.480 & 0.009 & 0.178 & 0.001 & 1.6 \\
$35_{d}$ & 108.92 & -0.417 & 0.009 & 0.164 & 0.001 & 1.6 \\
$32_{d}$ & 114.97 & -0.338 & 0.009 & 0.166 & 0.001 & 1.6 \\
$29_{d}$ & 121.03 & -0.219 & 0.009 & 0.172 & 0.001 & 1.7 \\
$26_{d}$ & 127.09 & -0.106 & 0.007 & 0.187 & 0.001 & 1.7 \\
$23_{d}$ & 133.17 & 0.019 & 0.007 & 0.216 & 0.001 & 1.7 \\
$20_{d}$ & 139.26 & 0.121 & 0.006 & 0.241 & 0.001 & 1.7 \\
$17_{d}$ & 145.36 & 0.195 & 0.006 & 0.257 & 0.002 & 1.7 \\
$14_{d}$ & 151.46 & 0.229 & 0.005 & 0.298 & 0.001 & 1.7 \\
$11_{d}$ & 157.57 & 0.201 & 0.005 & 0.371 & 0.001 & 1.7 \\
$9_{d}$ & 162.66 & 0.145 & 0.004 & 0.411 & 0.001 & 1.7 \\
$5_{d}$ & 169.80 & 0.067 & 0.005 & 0.553 & 0.001 & 1.7 \\
\hline \hline & & & & & & \\
\hline \hline
\end{tabular}

TABLE VI. Results for the vector analyzing power $A_{y}$ and the differential cross section $d \sigma / d \Omega$ at $190 \mathrm{MeV}$ incident-beam energy. The notation and the units are the same as given in Table I. The point-to-point uncertainty of the data, as discussed in Sec. IV D, results in a systematic uncertainty of $3 \%$, from which a total systematic uncertainty of $<4.5 \%$ is obtained. For the analyzing powers, a systematic uncertainty of around $3 \%$ should be taken for all the points except for the first seven and the last four points where the systematic uncertainty was around $4 \%$.

\begin{tabular}{|c|c|c|c|c|c|c|c|c|}
\hline$\theta_{\mathrm{lab}}$ & $\theta_{\text {c.m. }}$ & $A_{y}$ & $\Delta A_{y, S}$ & $d \sigma$ & $\Delta d \sigma_{S}$ & $\Delta d \sigma_{P}$ & $\Delta d \sigma_{N}$ & $\Delta d \sigma_{T}$ \\
\hline $17_{p}$ & 26.49 & 0.690 & 0.003 & 6.005 & 0.011 & 1.3 & 2.8 & 3.1 \\
\hline $20_{p}$ & 31.10 & 0.686 & 0.002 & 4.383 & 0.007 & 1.2 & 2.8 & 3.0 \\
\hline $23_{p}$ & 35.69 & 0.614 & 0.004 & 3.123 & 0.009 & 1.0 & 2.8 & 3.0 \\
\hline $26_{p}$ & 40.24 & 0.502 & 0.003 & 2.363 & 0.005 & 0.9 & 2.8 & 2.9 \\
\hline $29_{p}$ & 44.75 & 0.384 & 0.007 & 1.710 & 0.008 & 0.6 & 2.8 & 2.9 \\
\hline $32 p$ & 49.22 & 0.192 & 0.004 & 1.388 & 0.004 & 0.4 & 2.8 & 2.8 \\
\hline $35_{p}$ & 53.64 & 0.053 & 0.008 & 1.037 & 0.006 & 0.1 & 2.8 & 2.8 \\
\hline $38_{p}$ & 58.01 & -0.074 & 0.008 & 0.780 & 0.004 & 0.04 & 1.5 & 1.5 \\
\hline $41_{p}$ & 62.32 & -0.197 & 0.009 & 0.624 & 0.005 & 0.1 & 1.4 & 1.4 \\
\hline $44_{p}$ & 66.58 & -0.250 & 0.009 & 0.523 & 0.003 & 0.1 & 1.5 & 1.5 \\
\hline $47_{p}$ & 70.77 & -0.326 & 0.009 & 0.429 & 0.003 & 0.2 & 1.5 & 1.5 \\
\hline $50_{p}$ & 74.90 & -0.399 & 0.010 & 0.341 & 0.002 & 0.2 & 1.5 & 1.5 \\
\hline $50_{d}$ & 78.75 & -0.453 & 0.009 & 0.273 & 0.002 & 0.2 & 1.5 & 1.5 \\
\hline $47_{d}$ & 84.73 & -0.484 & 0.009 & 0.222 & 0.001 & 0.2 & 1.5 & 1.5 \\
\hline $44_{d}$ & 90.73 & -0.513 & 0.011 & 0.180 & 0.001 & 0.3 & 1.5 & 1.5 \\
\hline $41_{d}$ & 96.74 & -0.540 & 0.009 & 0.164 & 0.001 & 0.3 & 2.2 & 2.2 \\
\hline $38_{d}$ & 102.76 & -0.455 & 0.012 & 0.137 & 0.001 & 0.2 & 1.5 & 1.5 \\
\hline $35_{d}$ & 108.80 & -0.379 & 0.011 & 0.129 & 0.001 & 0.2 & 1.5 & 1.5 \\
\hline $32_{d}$ & 114.85 & -0.294 & 0.014 & 0.129 & 0.001 & 0.1 & 1.5 & 1.5 \\
\hline $29_{d}$ & 120.91 & -0.162 & 0.012 & 0.126 & 0.001 & 0.1 & 1.5 & 1.5 \\
\hline $26_{d}$ & 126.99 & -0.042 & 0.007 & 0.146 & 0.001 & 0.04 & 2.2 & 2.2 \\
\hline $23_{d}$ & 133.08 & 0.045 & 0.013 & 0.156 & 0.001 & 0.02 & 1.5 & 1.5 \\
\hline $20_{d}$ & 139.17 & 0.143 & 0.007 & 0.180 & 0.001 & 0.1 & 2.2 & 2.2 \\
\hline $17_{d}$ & 145.28 & 0.188 & 0.010 & 0.201 & 0.001 & 0.1 & 1.5 & 1.5 \\
\hline $14_{d}$ & 151.40 & 0.220 & 0.006 & 0.264 & 0.001 & 0.4 & 2.8 & 2.8 \\
\hline $11_{d}$ & 157.52 & 0.183 & 0.014 & 0.305 & 0.002 & 0.3 & 2.8 & 2.8 \\
\hline $9_{d}$ & 162.62 & 0.164 & 0.016 & 0.351 & 0.003 & 0.3 & 2.8 & 2.8 \\
\hline $5_{d}$ & 169.78 & 0.075 & 0.010 & 0.446 & 0.002 & 0.1 & 2.8 & 2.8 \\
\hline
\end{tabular}

[1] R. Machleidt, F. Sammarruca, and Y. Song, Phys. Rev. C 53, 1483(R) (1996).

[2] R. B. Wiringa, V. G. J. Stoks, and R. Schiavilla, Phys. Rev. C 51, 38 (1995).

[3] V. G. J. Stoks, R. A. M. Klomp, M. C. M. Rentmeester, and J. J. de Swart, Phys. Rev. C 48, 792 (1993).

[4] V. G. J. Stoks, R. A. M. Klomp, C. P. F. Terheggen, and J. J. de Swart, Phys. Rev. C 49, 2950 (1994).

[5] M. C. M. Rentmeester, R. G. E. Timmermans, J. L. Friar, and J. J. de Swart, Phys. Rev. Lett. 82, 4992 (1999).

[6] W. Glöckle, H. Witała, D. Hüber, H. Kamada, and J. Golak, Phys. Rep. 274, 107 (1996).
[7] H. Witała W. Glöckle, J. Golak, A. Nogga, H. Kamada, R. Skibinski, and J. Kuros-Zolnierczuk, Phys. Rev. C 63, 024007 (2001).

[8] H. Sakai et al., Phys. Rev. Lett. 84, 5288 (2000).

[9] K. Sekiguchi et al., Phys. Rev. C 65, 034003 (2002).

[10] H. Witała W. Glöckle, D. Hüber, J. Golak, and H. Kamada, Phys. Rev. Lett. 81, 1183 (1998).

[11] S. Nemoto, K. Chmielewski, S. Oryu, and P. U. Sauer, Phys. Rev. C 58, 2599 (1998) and references therein.

[12] H. Shimizu et al., Nucl. Phys. A382, 242 (1982).

[13] H. Postma and R. Wilson, Phys. Rev. 121, 1229 (1961). 
[14] K. Kuroda, A. Michalowicz, and M. Poulet, Nucl. Phys. 88, 33 (1966).

[15] G. Igo et al., Nucl. Phys. A195, 33 (1972).

[16] R. E. Adelberger and C. N. Brown, Phys. Rev. D 5, 2139 (1972).

[17] S. P. Wells et al., Nucl. Instr. and Meth. Phys. Res. A 325, 205 (1993).

[18] R. Bieber et al., Phys. Rev. Lett. 84, 606 (2000).

[19] R. V. Cadman et al., Phys. Rev. Lett. 86, 967 (2001).

[20] E. J. Stephenson H. Witala, W. Glöckle, H. Kamada, and A. Nogga, Phys. Rev. C 60, 061001(R) (1999).

[21] K. Ermisch et al., Phys. Rev. Lett. 86, 5862 (2001).

[22] K. Ermisch et al., Phys. Rev. C 68, 051001(R) (2003).

[23] D. Hüber, H. Kamada, H. Witała, and W. Glöckle, Acta Phys. Pol. B 28, 1677 (1997).

[24] A. Stadler, W. Glöckle, and P. U. Sauer, Phys. Rev. C 44, 2319 (1991).

[25] H. Witała, T. Cornelius, and W. Glöckle, Few Body Syst. 3, 123 (1988).

[26] D. Hüber, H. Witała, and W. Glöckle, Few Body Syst. 14, 171 (1993).

[27] S. A. Coon and H. Han, Few Body Syst. 30, 131 (2001).

[28] A. Nogga, H. Kamada, W. Glöckle, and B. R. Barrett, Phys. Rev. C 65, 054003 (2002).

[29] J. Carlson, V. R. Pandharipande, and R. B. Wiringa, Nucl. Phys. A401, 59 (1983).

[30] S. A. Coon et al., Nucl. Phys. A317, 242 (1979).

[31] J. L. Friar, D. Hüber, and U. van Kolck, Phys. Rev. C 59, 53 (1999).

[32] E. Epelbaum, Ph.D. thesis, Ruhr-Universität Bochum (2000).

[33] E. Epelbaum, W. Glöckle, and U.-G. Meißner, Nucl. Phys. A671, 295 (2000).

[34] U. van Kolck, Phys. Rev. C 49, 2932 (1994).

[35] E. Epelbaum, A. Nogga, W. Glöckle, H. Kamada, Ulf-G. Meißner, and H. Witała, Phys. Rev. C 66, 064001 (2002).

[36] E. Epelbaum, A. Nogga, W. Glöckle, H. Kamada, U.-G. Meißner, and H. Witała, Eur. Phys. J. A 15, 543 (2002).

[37] E. Epelbaum, W. Glöckle, and U.-G. Meißner, Eur. Phys. J. A 19, 125 (2004).

[38] E. Epelbaum, W. Glöckle, and U.-G. Meißner, Eur. Phys. J. A 19, 401 (2004).
[39] E. Epelbaum et al., in preparation.

[40] A. Deltuva, K. Chmielewski, and P. U. Sauer, Phys. Rev. C 67, 034001 (2003).

[41] R. Machleidt, Phys. Rev. C 63, 024001 (2001).

[42] A. Deltuva, R. Machleidt, and P. U. Sauer, Phys. Rev. C 68, 024005 (2003).

[43] J. Fujita and H. Miyazawa, Progr. Theor. Phys. 17, 360 (1957).

[44] S. C. Pieper, V. R. Pandharipande, R. B. Wiringa, and J. Carlson, Phys. Rev. C 64, 014001 (2001).

[45] E. O. Alt, P. Grassberger, and W. Sandhas, Nucl. Phys. B2, 167 (1967).

[46] L. Friedrich, E. Huttel, R. Kremers, and A. G. Drentje, in Proceedings of the International Workshop on Polarized Beams and Polarized Targets, Köln, 1995, edited by H. P. gen Schieck and L. Sydow (World Scientific, Singapore, 1996), p. 198.

[47] R. Bieber et al., Nucl. Instr. and Meth. Phys. Res. A 457, 12 (2001).

[48] A. M. van den Berg, Nucl. Instr. and Meth. Phys. Res. B 99, 637 (1995).

[49] H. J. Wörtche, Nucl. Phys. A687, 321c (2001).

[50] K. Ermisch, Ph.D. thesis, Rijksuniversiteit Groningen (2003).

[51] Online calculations from nijmegen potentials and partial-wave analysis, nn-online.sci.kun.nl.

[52] R. Timmermans, private communication (2002).

[53] G. C. Salzmann, C. K. Mitchell, and G. G. Ohlson, Nucl. Instrum, Meth. 109, 61 (1973).

[54] F. Zwarts, KVI Annual Report p. 89 (1996).

[55] V. M. Hannen, Ph.D. thesis, Rijksuniversiteit Groningen (2001).

[56] P. Kuipers-Munneke, internal report (2002).

[57] H. A. J. Meijer, B. A. M. Kers, E. Kerstel, and P. KuipersMunneke, private communication (2002).

[58] F. Hinterberger, private communication (2002).

[59] G. G. Ohlson, Rep. Prog. Phys. 35, 717 (1972).

[60] P. C. Gugelot, J. Källne, and P. U. Renberg, Phys. Scr. 10, 252 (1974).

[61] B. V. Przewoski, Phys. Rev. C (to be published), nuclex/0411019 (2004).

[62] M. Volkerts, Ph.D. thesis, Rijksuniversiteit Groningen (2001).

[63] M. Garçon et al., Nucl. Phys. A458, 287 (1986).

[64] H. Kamada W. Glöckle, J. Golak, and Ch. Elster, Phys. Rev. C 66, 044010 (2002). 